NASA Technical Memorandum 100875

ICOMP-88-10

\title{
Solving Time-Dependent Two-Dimensional Eddy Current Problems
}

Min Eig Lee

University of Akron

Akron, Ohio

and

S.I. Hariharan

Institute for Computational Mechanics in Propulsion

Lewis Research Center

Cleveland, Ohio

and

Nathan Ida

University of Akron

Akron, Ohio

June 1988

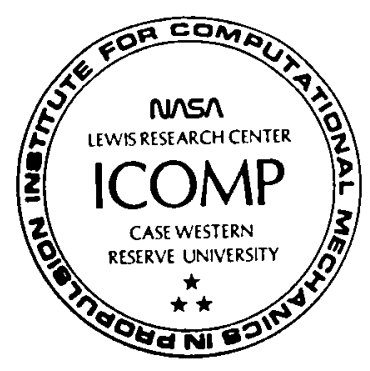




\title{
Solving Time-Dependent Two-Dimensional Eddy Current Problems
}

\author{
Min Eig Lee \\ Department of Electrical Engineering, \\ University of Akron, Akron, Ohio 44325 \\ S.I. Hariharan* \\ Department of Mathematical Sciences, \\ University of Akron, Akron, Ohio 44325, \\ and Institute for Computational Mechanics in Propulsion, \\ Lewis Research Center, Cleveland, Ohio 44135 \\ and \\ Nathan Ida \\ Department of Electrical Engineering, \\ University of Akron, Akron, Ohio 44325
}

\section{Introduction}

Eddy current calculation is an important subject in classical electrodynamics. Eddy currents are induced in a conductor, when an electromagnetic field is incident upon it. Usually these phenomena result in flow of currents in a region near the boundary. A common way of treating these problems, in particular these induced currents, relies on estimating an appropriate skin depth on the conductor. Once the skin depth is determined, the associated fields are obtained through an equivalence principle by imposing a boundary condition on the region of current formulation. Such a treatment may be found in [12]. The estimated skin depth (given at the end of the paper) is obtained by the penetration of fields into an infinite planar slab. However, if the conductor happens to have geometry other than that of a planar slab, the procedure mentioned above will not work well. Moreover, the incident waves need not be restricted to time-harmonic waves in general. Pulse sources and surge waves which come from thunder or circuit breakers

\footnotetext{
"Work was supported by National Science Foundation Grant DMS-8604047, and in part by NASA (funded under Space Act Agreement C99066G) while the author was visiting ICOMP, NASA Lewis Research Center.
} 
arise in practice. Therefore, one must have a procedure which reflects the transient behavior of eddy currents.

Along this line, an effort was begun in [1], in which a frequency domain problem was considered. This procedure presented some possibilities for further improvements and the feasibility of solving this class of problems in three dimensions. The work in [3] dealt with continuation of [1] in conjunction with finite element methods. Unfortunately, only theoretical details are available in this work. Moreover, the treatment in the threedimensional problem was restricted to time-harmonic fields. However, it was established in [4] and [5] that the finite difference methods are effective even for frequency domain problems, i. e. , pseudo-time marching. Our goal is to use finite difference methods to solve these problems, in particular, the two-dimensional problem in the time domain. In the engineering literature, for example, in [6], the author pointed out the computational difficulties in the problem. To motivate the ideas, the problem under consideration is presented in the next section.

\section{Formulation of the Problem}

Let $\Omega$ be the cross-section region in the x-y plane of a conducting cylinder with finite conductivity $\sigma_{m}$, permeability $\mu_{m}$, and permittivity $\epsilon_{m}$. The generators of the cylinder are parallel to the $z$ axis. The ambient medium is air with permeability $\mu_{a}$ and permittivity $\epsilon_{a}$. The conductivity of the air is neglected because it does not play a major role in this type of calculations. If desired, it could be incorporated into this model, in which case the following treatment would still be valid. The incident field has the form

$$
\begin{aligned}
\mathbf{E}_{i} & =E_{i}(x, y, t) \mathbf{k} \\
\mathrm{H}_{i} & =H_{i 1}(x, y, t) \mathbf{i}+H_{i 2}(x, y, t) \mathbf{j} .
\end{aligned}
$$

A typical situiation is depicted in Fig. 1. The electric field is parallel to the axis of the cylinder and the magnetic field is transverse (TM) and in the

$\mathrm{x}-\mathrm{y}$ plane. Writing Maxwell's equations, assuming all material properties are constant and isotropic, one obtains two sets of equations that are valid inside and outside the conductor respectively.

In $\Omega_{A}$ (Air)

$$
\frac{\partial H_{1}}{\partial x}+\frac{\partial H_{2}}{\partial y}=0
$$




$$
\begin{aligned}
\frac{\partial E}{\partial y} \mathbf{i}-\frac{\partial E}{\partial x} \mathbf{j} & =-\mu_{a}\left(\frac{\partial H_{1}}{\partial t} \mathbf{i}+\frac{\partial H_{2}}{\partial t} \mathbf{j}\right), \\
\frac{\partial H_{2}}{\partial x}-\frac{\partial H_{1}}{\partial y} & =\epsilon_{a} \frac{\partial E}{\partial t}
\end{aligned}
$$

\section{In $\Omega$ (Conductor)}

$$
\begin{aligned}
& \frac{\partial H_{1}}{\partial x}+\frac{\partial H_{2}}{\partial y}=0, \\
& \frac{\partial E}{\partial y} \mathbf{i}-\frac{\partial E}{\partial x} \mathbf{j}=-\mu_{m}\left(\frac{\partial H_{1}}{\partial t} \mathbf{i}+\frac{\partial H_{2}}{\partial t} \mathbf{j}\right), \\
& \frac{\partial H_{2}}{\partial x}-\frac{\partial H_{1}}{\partial y}=\sigma_{m} E+\epsilon_{m} \frac{\partial E}{\partial t}
\end{aligned}
$$

The first term on the right side of $(8)$ is the conduction current and the second term is the displacement current. In addition, the following conditions are needed to make the problem well-posed.

On $\Gamma$ (Boundary of $\Omega$ ) Tangential components of $E$ and $H$ are continuous, i.e., no surface current density is assumed.

At infinity the scattered $\mathbf{E}$ and $\mathbf{H}$ both decay to zero (Radiation Condition).

The zero divergence and two-dimensionality of $\mathrm{H}$ in both regions suggest the existence of a scalar function $\Psi$ such that

$$
\begin{aligned}
& H_{1}=\frac{\partial \Psi}{\partial y}, \\
& H_{2}=-\frac{\partial \Psi}{\partial x} .
\end{aligned}
$$

Substituting these in equations (4) and (7), we see that

$$
E=-\mu \frac{\partial \Psi}{\partial t} \text {. }
$$

The function $\mu \Psi \mathrm{k}$ is identified as the magnetic vector potential. In turn, using this scalar function, the problem can be rewritten as follows. 
The governing equations are

$$
\begin{array}{ll}
\frac{\partial^{2} \Psi}{\partial x^{2}}+\frac{\partial^{2} \Psi}{\partial y^{2}}=\epsilon_{a} \mu_{a} \frac{\partial^{2} \Psi}{\partial t^{2}} & \text { in } \Omega_{A}, \\
\frac{\partial^{2} \Psi}{\partial x^{2}}+\frac{\partial^{2} \Psi}{\partial y^{2}}=\sigma_{m} \mu_{m} \frac{\partial \Psi}{\partial t}+\epsilon_{m} \mu_{m} \frac{\partial^{2} \Psi}{\partial t^{2}} & \text { in } \Omega .
\end{array}
$$

The interface conditions on $\Gamma$ are

$$
\begin{aligned}
\mu_{a}\left(\frac{\partial \Psi}{\partial t}\right)^{-} & =\mu_{m}\left(\frac{\partial \Psi}{\partial t}\right)^{+} \\
\left(\frac{\partial \Psi}{\partial n}\right)^{-} & =\left(\frac{\partial \Psi}{\partial n}\right)^{+},
\end{aligned}
$$

where the minus sign indicates transition from the exterior to the boundary and the plus sign indicates transition from the boundary to the interior $\Omega$. Since the problem is posed in the time domain, we require appropriate initial conditions.

The initial conditions are

$$
\begin{aligned}
\Psi(x, y, 0) & =\Psi_{i}(x, y, 0) \\
\frac{\partial \Psi(x, y, 0)}{\partial t} & =\frac{\partial \Psi_{i}(x, y, 0)}{\partial t}
\end{aligned}
$$

Sommerfeld's radiation condition is

$$
\lim _{r \rightarrow \infty} r^{1 / 2}\left(\frac{\partial \Psi_{s}}{\partial r}+\sqrt{\mu_{a} \epsilon_{a}} \frac{\partial \Psi_{s}}{\partial t}\right)=0
$$

where $\Psi_{s}$ represents the scattered wave.

\section{Difficulties in the Problem}

The problem presented in equations (12) through (18) yields an interface problem with $\Gamma$ being the interface. This model is also applicable in calculating wave scattering by lossy dielectrics and the nondestructive evaluation(NDE) of various kinds of composite materials. In the event of composite materials, $\mu_{m}$ and $\epsilon_{m}$ will no longer be constants. Rather, they will be functions of space. The frequency of the incident wave may range from low frequencies to microwave frequencies.

In solving this problem, one encounters certain difficulties. They are highlighted as follows: 
1. Implementation of the radiation condition.

2. Implementation of the interface conditions.

3. The large number of grid points that is needed to capture the skin depth in the high-frequency case.

4. Appropriate local boundary condition for the low-frequency cases, where the wavelengths are much longer than the diameter of the conductor.

To further address and answer the difficulties, we require scaling of the problem. Our scaling is performed as follows.

1. Scale $\Psi$ by $\Psi_{0}$, i. e. , $\Psi=\Psi / \Psi_{0}$, where $\Psi_{0}$ is the amplitude of the incident wave $\Psi_{i}$.

2. Scale $\mathrm{x}$ and $\mathrm{y}$ by $\mathrm{L}$, i. e. , $\bar{x}=x / L, \bar{y}=y / L$, where $\mathrm{L}$ is a length of the order of the scatterer's diameter.

3. Scale t by $1 / \omega$, i. e. , $\bar{t}=\omega t$, where $\omega$ is the angular velocity of the incident wave.

As a result, the problem in these new coordinates has the following form:

\section{Governing Equations}

$$
\begin{aligned}
& \frac{\partial^{2} \bar{\Psi}}{\partial \bar{x}^{2}}+\frac{\partial^{2} \bar{\Psi}}{\partial \bar{y}^{2}}=\omega^{2} L^{2} \mu_{a} \epsilon_{a} \frac{\partial^{2} \bar{\Psi}}{\partial \bar{t}^{2}} \\
& \frac{\partial^{2} \bar{\Psi}}{\partial \bar{x}^{2}}+\frac{\partial^{2} \bar{\Psi}}{\partial \bar{y}^{2}}=\omega L^{2} \mu_{m} \sigma_{m} \frac{\partial \bar{\Psi}}{\partial \bar{t}}+\omega^{2} L^{2} \mu_{m} \epsilon_{m} \frac{\partial^{2} \bar{\Psi}}{\partial \bar{t}^{2}}
\end{aligned}
$$

Interface Conditions

$$
\begin{aligned}
& \mu_{a} \bar{\Psi}=\mu_{m} \bar{\Psi}^{+} \\
& \frac{\partial \bar{\Psi}}{\partial \bar{n}}=\frac{\partial \bar{\Psi}^{+}}{\partial \bar{n}}
\end{aligned}
$$

Initial Conditions

$$
\begin{aligned}
\bar{\Psi}(\bar{x}, \bar{y}, 0) & =\overline{\Psi_{i}}(\bar{x}, \bar{y}, 0) \\
\frac{\partial \bar{\Psi}}{\partial \bar{t}}(\bar{x}, \bar{y}, 0) & =\frac{\partial \overline{\Psi_{i}}}{\partial \bar{t}}(\bar{x}, \bar{y}, 0) .
\end{aligned}
$$




\section{Radiation Condition}

$$
\lim _{\bar{r} \rightarrow \infty} \bar{r}^{1 / 2}\left(\frac{\partial \overline{\Psi_{s}}}{\partial \bar{r}}+k_{a} \frac{\partial \overline{\Psi_{s}}}{\partial \bar{t}}\right)=0,
$$

where $k_{a}=\omega L \sqrt{\mu_{a} \epsilon_{a}}$.

The difficulty with these problems can be simply explained now. For instance, if we consider a $60 \mathrm{~Hz}$ incident field and a conductor with a diameter of $.3141593 \mathrm{~m}$, the parameters that appear in equations (19) and (20) have the following values: $k_{a}=\omega L \sqrt{\mu_{a} \epsilon_{a}}=2.513274 \times 10^{-7}$, $k_{m}^{\prime}=\omega L \sqrt{\mu_{m} \epsilon_{m}}=2.513274 \times 10^{-7}, l_{m}^{\prime}=L \sqrt{\omega \mu_{m} \epsilon_{m}}=26.55045$, where we have used: $\mu_{a}=\mu_{m}=1.256637 \times 10^{-6}, \epsilon_{a}=\epsilon_{m}=8.841941 \times 10^{-12}$, $\sigma_{m}=3.72 \times 10^{7}$, and $L=0.2$ in the MKS unit system. Thus, reconsidering equation (19), we see that it behaves like Laplace's equation, while equation (20) behaves like a parabolic equation. These observations were made in [13]. This only yields an approximation to the model given in equations (19) through (25). We believe that even though the value of $k_{a}$ is extremely small, the problem is still governed by a wave phenomenon. This is further justified by the results that we present later. Thus, we do not approximate the model, but rather present procedures to solve the problem as we have derived. This yields several difficulties, particularly in terms of the computation. Our treatment is presented in the coming sections.

Thus retaining the wave nature in the exterior, we further scale the problem for the purpose of treatment of boundary conditions. We scale the time by $k_{a}$. The result yield the following nondimensional problem that we numerically solve:

\section{Governing Equations}

$$
\begin{aligned}
& \frac{\partial^{2} \Psi}{\partial x^{2}}+\frac{\partial^{2} \Psi}{\partial y^{2}}=\frac{\partial^{2} \Psi}{\partial t^{2}} \\
& \frac{\partial^{2} \Psi}{\partial x^{2}}+\frac{\partial^{2} \Psi}{\partial y^{2}}=l_{m}^{2} \frac{\partial \Psi}{\partial t}+k_{m}^{2} \frac{\partial^{2} \Psi}{\partial t^{2}}
\end{aligned}
$$

where

$$
l_{m}^{2}=\frac{\omega L^{2} \mu_{m} \sigma_{m}}{k_{a}},
$$

and

$$
k_{m}^{2}=\frac{\omega^{2} L^{2} \mu_{m} \epsilon_{m}}{k_{a}^{2}}
$$


Interface Conditions

$$
\begin{aligned}
\mu_{a} \Psi^{-} & =\mu_{m} \Psi^{+} \\
\frac{\partial \Psi^{-}}{\partial n} & =\frac{\partial \Psi^{+}}{\partial n}
\end{aligned}
$$

Initial Conditions

$$
\begin{aligned}
\Psi(x, y, 0) & =\Psi_{i}(x, y, 0) \\
\frac{\partial \Psi}{\partial t}(x, y, 0) & =\frac{\partial \Psi_{i}}{\partial t}(x, y, 0) .
\end{aligned}
$$

\section{Radiation Condition}

$$
\lim _{r \rightarrow \infty} r^{1 / 2}\left(\frac{\partial \Psi_{s}}{\partial r}+\frac{\partial \Psi_{s}}{\partial t}\right)=0
$$

Note that the bar notation has been dropped.

\section{Radiation Condition}

The radiation condition as given in equation (32) is hard to implement numerically. It must be approximated in a suitable way before it is imposed on a computation domain. For the waves with moderate to high frequencies, the forms of this condition are well known. A summary may be found in [7]. The procedure here is to write the far-field scattered wave in the form:

$$
\Psi_{s}=\frac{f\left(k_{a}(r-t)\right)}{\sqrt{r}}\left(a_{0}(\theta)+\frac{a_{1}(\theta)}{r}+\frac{a_{2}(\theta)}{r^{2}}+\cdots\right) .
$$

This expression is the extended D'Alembert's principle and indicates the fact that all the scattered fields are outgoing. From this, as in [7], it is easily verified that $\Psi_{s}$ satisfies

$$
\frac{\partial \Psi_{s}}{\partial r}+\frac{\partial \Psi_{s}}{\partial t}+\frac{1}{2 r} \Psi_{s}=O\left(\frac{1}{r^{5 / 2}}\right) .
$$

However, for low-frequency waves, equation (34) does not provide satisfactory solutions. This is due to the fact that the wavelengths are very long and the substantial distances for the far-field boundaries require at least one wavelength. This is not practical for numerical implementation. Thus 
we introduce a new concept of formulating near-field conditions. Again the procedure is analogous to the one used in (33) and (34), but equation (33) will be replaced by an outgoing near-field solution.

As suggested in [2], for low-frequency waves, the solution is dominated by logarithmic terms in both space and wave number. Thus one can seek solutions of the form:

$$
\Psi_{s} \approx(a+b \ln r) f\left(k_{a}(r-t)\right)
$$

where $\mathrm{a}, \mathrm{b}$ are constants. From this one obtains

$$
\frac{\partial \Psi_{s}}{\partial r}+\frac{\partial \Psi_{s}}{\partial t}+A(r) \Psi_{s} \approx 0
$$

where

$$
A(r)=\frac{1}{r}\left(-\frac{b}{a+b \ln r}\right)
$$

For low-frequency time-harmonic waves,

$$
a \approx b\left(\gamma+\ln \frac{k_{a}}{2}\right)
$$

where $\gamma$ is Euler's constant. Substituting in A(r), we obtain

$$
A(r)=\frac{1}{r}\left[-\frac{1}{\gamma-\ln 2+\ln k_{a}+\ln r}\right] .
$$

It is easy to verify that $A(r)$ is positive in the low-frequency case.

With equations (34) and (36) imposed on the exterior boundary, the problem becomes well-posed. The proof for the uniqueness of the solution is shown in the appendix $\mathrm{A}$.

\section{Finite Difference Formulation of the Problem}

The finite difference method has been found effective in time dependent hyperbolic problems. In addition, the radiation conditions in the previous section can easily be integrated in the scheme. Finite difference formulae used in solving this problem are summarized as follows. Note that the spatial differences in both $\mathrm{x}$ and $\mathrm{y}$ directions are chosen to be equal, i. e. , $\Delta x=\Delta y$. The superscript ' $\mathrm{i}$ ' represents the time coordinate and the subscripts ' $\mathrm{j}$ ' and ' $\mathrm{k}$ ' represent the grid coordinates in the $\mathrm{x}$ and $\mathrm{y}$ directions respectively. 
In the exterior region, the following central-difference formula is used to discretize equation (26):

$\Psi_{j, k}^{i+1}=2 \Psi_{j, k}^{i}-\Psi_{j, k}^{i-1}+\left(\frac{\Delta t}{\Delta x}\right)^{2}\left(\Psi_{j+1, k}^{i}+\Psi_{j-1, k}^{i}+\Psi_{j, k+1}^{i}+\Psi_{j, k-1}^{i}-4 \Psi_{j, k}^{i}\right)$.

In the interior region, the first-order time derivative $\partial \Psi / \partial t$ is implemented by a forward-difference method. The second-order derivatives are implemented by central-difference methods. As the result, the following difference formula is obtained for Eq. (27):

$$
\Psi_{j, k}^{i+1}=a \Psi_{j, k}^{i}-b \Psi_{j, k}^{i-1}+c\left(\Psi_{j+1, k}^{i}+\Psi_{j-1, k}^{i}+\Psi_{j, k+1}^{i}+\Psi_{j, k-1}^{i}-4 \Psi_{j, k}^{i}\right) .
$$

where

$$
\begin{aligned}
& a=\frac{2+\frac{l_{m}^{2}}{k_{m}^{2}} \Delta t}{1+\frac{l_{m}^{2}}{k_{m}^{2}} \Delta t}, \\
& b=\frac{1}{1+\frac{l_{m}^{2}}{k_{m}^{2}} \Delta t}, \\
& c=\frac{\frac{1}{k_{m}^{2}}\left(\frac{\Delta t}{\Delta x}\right)^{2}}{1+\frac{l_{m}^{2}}{k_{m}^{2}} \Delta t} .
\end{aligned}
$$

On the radiation boundary, the total wave consists of two components: the incident wave $\Psi_{i}$ and the scattered wave $\Psi_{s}$, i. e. ,

$$
\Psi=\Psi_{i}+\Psi_{s}
$$

For the far field, the scattered wave propagates in a direction which is very close to the radial direction. The origin is located at the center of the scatterer as shown in Fig. 2. Using the derivatives in Cartesian coordinates, the radiation condition has the following form:

$$
\frac{\partial \Psi_{s}}{\partial x} \cos \theta+\frac{\partial \Psi_{s}}{\partial y} \sin \theta+\frac{\partial \Psi_{s}}{\partial t}+\frac{\Psi_{s}}{2 r}=0
$$

Finite difference methods usually yield difficulties at the corners of the rectangular radiation boundary. These are due to conflicts between the difference formulae at the corners. As a result, these corners become sources of instability. To avoid corner problems, Engquist and Majda in [8] proposed 
a different boundary condition be used for the nearest two grid points to the corners. Unfortunately in this way, the implementation of the radiation boundary condition becomes more complicated. In our experiment, a method using a smooth transition was developed to solve corner problems. The approach is depicted in Fig. 3 and explained as follows. On the boundary $A_{1}$ to $A_{2}, \partial \Psi_{s} / \partial x$ is implemented by a forward-difference formula and $\partial \Psi_{s} / \partial y$ is implemented by a backward-difference formula. On the boundary $B_{1}$ to $B_{2}, \partial \Psi_{s} / \partial x$ and $\partial \Psi_{s} / \partial y$ are both implemented by backward-difference methods. It seems that implementation of $\partial \Psi_{s} / \partial x$ on two boundaries will conflict at the intersection grid C. But since the term is multiplied by $\cos \theta$ and its value is 0 at $C$, the conflict can not take effect and corner problems are avoided.

The difference formulae on the radiation boundary are summarized as follows.

At $A_{0}$

$$
\begin{aligned}
\left(\Psi_{s}\right)_{j, k}^{i+1} & =a\left(\Psi_{s}\right)_{j, k}^{i}-b\left[\left(\Psi_{s}\right)_{j+1, k}^{i}-\left(\Psi_{s}\right)_{j, k}^{i}\right] \\
\Psi_{j, k}^{i+1} & =\left(\Psi_{i}\right)_{j, k}^{i+1}+\left(\Psi_{s}\right)_{j, k}^{i+1}
\end{aligned}
$$

On $A_{1}-A_{2}$

$$
\begin{aligned}
\left(\Psi_{s}\right)_{j, k}^{i+1}= & a\left(\Psi_{s}\right)_{j, k}^{i}-b\left[\left(\Psi_{s}\right)_{j+1, k}^{i}-\left(\Psi_{s}\right)_{j, k}^{i}\right] \\
& -c\left[\left(\Psi_{s}\right)_{j, k}^{i}-\left(\Psi_{s}\right)_{j, k-1}^{i}\right] \\
\Psi_{j, k}^{i+1}= & \left(\Psi_{i}\right)_{j, k}^{i+1}+\left(\Psi_{s}\right)_{j, k}^{i+1}
\end{aligned}
$$

At $\mathrm{C}$

$$
\begin{aligned}
\left(\Psi_{s}\right)_{j, k}^{i+1} & =a\left(\Psi_{s}\right)_{j, k}^{i}-c\left[\left(\Psi_{s}\right)_{j, k}^{i}-\left(\Psi_{s}\right)_{j, k-1}^{i}\right] \\
\Psi_{j, k}^{i+1} & =\left(\Psi_{i}\right)_{j, k}^{i+1}+\left(\Psi_{s}\right)_{j, k}^{i+1}
\end{aligned}
$$

On $B_{1}-B_{2}$

$$
\begin{aligned}
\left(\Psi_{s}\right)_{j, k}^{i+1}= & a\left(\Psi_{s}\right)_{j, k}^{i}-b\left[\left(\Psi_{s}\right)_{j, k}^{i}-\left(\Psi_{s}\right)_{j-1, k}^{i}\right] \\
& -c\left[\left(\Psi_{s}\right)_{j, k}^{i}-\left(\Psi_{s}\right)_{j, k-1}^{i}\right] \\
\Psi_{j, k}^{i+1}= & \left(\Psi_{i}\right)_{j, k}^{i+1}+\left(\Psi_{s}\right)_{j, k}^{i+1}
\end{aligned}
$$


At $B_{0}$

$$
\begin{aligned}
\left(\Psi_{s}\right)_{j, k}^{i+1} & =a\left(\Psi_{s}\right)_{j, k}^{i}-b\left[\left(\Psi_{s}\right)_{j, k}^{i}-\left(\Psi_{s}\right)_{j-1, k}^{i}\right] \\
\Psi_{j, k}^{i+1} & =\left(\Psi_{i}\right)_{j, k}^{i+1}+\left(\Psi_{s}\right)_{j, k}^{i+1}
\end{aligned}
$$

Conditions on the lower boundary are handled by symmetry as the upper boundary. The coefficients $a, b$, and $c$ are obtained in the following way:

$$
\begin{aligned}
& a=1-\frac{\Delta t}{2 r \Delta x}, \\
& b=\cos \theta \frac{\Delta t}{\Delta x}, \\
& c=\sin \theta \frac{\Delta t}{\Delta x} .
\end{aligned}
$$

The implementation of equation (36) is similar to the above.

In the neighborhood of the interface, numerical implementation of the interface conditions is complicated. In reference [11], interface conditions are implemented by a cell integration method. Here we will propose a simple way to solve it directly using the finite difference method.

First, for an interface boundary with some extent of curvature, we can approximate it by a polygon with its vertexes occupying the regular grids. For example, the circle $\Gamma$ in Fig. 4 can be approximated by the polygon $\Gamma^{\prime}$. Then the field computation can be classified into two categories.

1. For grids in the exterior region, the wave equation in the air is used.

2. For grids in the interior region, the wave equation inside the conductor is used.

For grids on the boundary, to obtain an accurate result, the following are suggested:

1. When the grid separation is much larger than the estimated skin depth, the interior wave equation is used.

2. When the grid separation is equal to or smaller than the estimated skin depth, the exterior wave equation is used.

Secondly, the condition

$$
\frac{\partial \Psi^{-}}{\partial n}=\frac{\partial \Psi^{+}}{\partial n}
$$


can be transformed into:

$$
\frac{\partial \Psi^{-}}{\partial x} \cos \theta+\frac{\partial \Psi^{-}}{\partial y} \sin \theta=\frac{\partial \Psi^{+}}{\partial x} \cos \theta+\frac{\partial \Psi^{+}}{\partial y} \sin \theta
$$

where $\theta$ is the angle between the normal direction and the $\mathrm{x}$ axis. Since $\cos \theta$ and $\sin \theta$ are linearly independent, we have the following relations on the interface boundary:

$$
\begin{aligned}
& \frac{\partial \Psi^{-}}{\partial x}=\frac{\partial \Psi^{+}}{\partial x} \\
& \frac{\partial \Psi^{-}}{\partial y}=\frac{\partial \Psi^{+}}{\partial y} .
\end{aligned}
$$

With these, when the field computation involves a grid in the other region, an equivalent value at that grid can be obtained for computation. For example , the field computation at the grid $\mathrm{A}$ in Fig. 5 belongs to the category of the air. But a grid B in the conductor is involved in the difference formula for $\mathrm{A}$ as shown below:

$$
\Psi_{A}^{i+1}=2 \Psi_{A}^{i}-\Psi_{A}^{i-1}+\left(\frac{\Delta t}{\Delta x}\right)^{2}\left(\left(\Psi_{B}\right)^{i}+\Psi_{C}^{i}+\Psi_{D}^{i}+\Psi_{E}^{i}-4 \Psi_{A}^{i}\right),
$$

where $\Psi_{B}^{-}$is the equivalent value of $\Psi^{-}$at $B$. It can be obtained in the following way:

$$
\Psi_{B}^{-}=\Psi_{B}^{+}+\left(1-1 / \mu_{r}\right) \Psi_{A}
$$

Substituting into the equation above, we obtain

$$
\Psi_{A}^{i+1}=2 \Psi_{A}^{i}-\Psi_{A}^{i-1}+\left(\frac{\Delta t}{\Delta x}\right)^{2}\left(\left(\Psi_{B}^{+}\right)^{i}+\Psi_{C}^{i}+\Psi_{D}^{i}+\Psi_{E}^{i}-\left(3+1 / \mu_{r}\right) \Psi_{A}^{i}\right)
$$

Similarly, using the first interface condition, the field at the grid 1 can be calculated from the following:

$$
\Psi_{1}^{i+1}=a \Psi_{1}^{i}-b \Psi_{1}^{i-1}+c\left(\left(\Psi_{2}^{-}\right)^{i} / \mu_{r}+\left(\Psi_{3}^{-}\right)^{i} / \mu_{r}+\Psi_{4}^{i}+\Psi_{5}^{i}-4 \Psi_{1}^{i}\right),
$$

where $a, b$, and $c$ are the same constants as those in equation (41).

When the relative permeability $\mu_{r}$ of the conductor is 1 , approximating the interface boundary by some other geometry is not necessary. The field computation is classified in the same way as above. When a field computation involves a grid in the other set, the field at that grid will be directly 
used for calculation because it equals to the equivalent value. For example, in Fig. 6, from the interface conditions,

$$
\begin{aligned}
\Psi_{B}^{-} & =\Psi_{B}^{+}, \\
\Psi_{C}^{-}-\Psi_{B}^{-} & =\Psi_{C}^{+}-\Psi_{B}^{+} .
\end{aligned}
$$

Thus $\Psi_{C}^{-}=\Psi_{C}^{+}$. As a result, the field at A can be obtained from the following formula:

$$
\Psi_{A}^{i+1}=2 \Psi_{A}^{i}-\Psi_{A}^{i-1}+\left(\frac{\Delta t}{\Delta x}\right)^{2}\left(\Psi_{C}^{i}+\Psi_{D}^{i}+\Psi_{E}^{i}+\Psi_{F}^{i}-4 \Psi_{A}^{i}\right)
$$

Similarly, the field at the grid 1 can be calculated from the following:

$$
\Psi_{1}^{i+1}=a \Psi_{1}^{i}-b \Psi_{1}^{i-1}+c\left(\Psi_{2}^{i}+\Psi_{3}^{i}+\Psi_{4}^{i}+\Psi_{5}^{i}-4 \Psi_{1}^{i}\right) .
$$

\section{$6 \quad$ Numerical Experiments}

The numerical methods presented above were implemented in a computer program running on a SUN4/260 workstation. The conductor is a circular cylinder and is assumed to be aluminum. The grid coordinates with useful parameters are shown in Fig. 7. The incident wave is assumed to be a sinusoidal TM plane wave propagating in the $\mathrm{x}$ direction. The wave is assumed to be incident on the left boundary starting at $t=0$. Therefore, the incident component at the grid point $(j, k)$ in the application of the radiation condition is

$$
\left(\Psi_{i}\right)_{j, k}^{i}=\cos \left[k_{a}(i \Delta t-j \Delta x)\right],
$$

where $k_{a}=\omega L \sqrt{\mu_{a} \epsilon_{a}}$. The reason for this is that the incident wave which arrives at the point $(\mathrm{j}, \mathrm{k})$ is the incident wave at the point $(0, \mathrm{k}), j \Delta x$ time units earlier.

The high frequency used in the numerical experiment is $2.387324 \times 10^{8}$ Hz. With this frequency, parameters have the following values:

- $k_{a}=1$

- $l_{m}^{2}=2.804814 \times 10^{9}$

- $k_{m}^{2}=1$. 
The numerical solution shown in Fig. 8 was obtained after ten periods from the beginning. The CPU time is 74 seconds. From the history plot at a point $(67,34)$ (see Fig. 13), the solution is found to be at steady state.

The low frequency used in the experiment is $60 \mathrm{~Hz}$. With this frequency, parameters have the following values:

- $k_{a}=2.513274 \times 10^{-7}$

- $l_{m}^{2}=2.804814 \times 10^{9}$

- $k_{m}^{2}=1$.

The numerical solution using the near-field condition(see Fig. 10), is obtained after $2.513274 \times 10^{-4}$ period. The CPU time is about $1.7 \mathrm{hrs}$. From the history plot at a point $(67,34)$ (see Fig. 14$)$, the solution in the exterior region is found to be in transient state but with a value almost approaching that at steady state. For reasons of comparison, the numerical solution using the far-field condition is also shown in Fig. 11.

\section{Comparison of Numerical and Analytic Solu- tion}

Since aluminum is a good conductor, In the high-frequency case, conductors such as aluminum behave close to a perfect conductor $\left(\sigma_{m}=\infty\right)$. The analytic solution for the perfect-conductor problem is found in the literature, [9] and [10], as follows:

$$
\begin{aligned}
\Psi^{-}\left(r, \phi, t^{\prime}\right) & =\sum_{n=0}^{\infty} i^{n} \varepsilon_{n} \cos (n \phi)\left[J_{n}\left(k_{a} r\right)-\frac{J_{n}\left(k_{a} a\right)}{I I_{n}^{(1)}\left(k_{a} a\right)} I_{n}^{(1)}\left(k_{a} r\right)\right] e^{-i k_{a} t^{\prime}} \\
\Psi^{+}\left(r, \phi, t^{\prime}\right) & =0
\end{aligned}
$$

where $J_{n}$ is the Bessel function of first kind and order $n, I_{n}^{(1)}$ is the Irankel function of first kind and order n. $\varepsilon_{0}=1$ and $\varepsilon_{n}=2$ for $n \geq 1$. This solution assumes a circular cylinder of radius a. With respect to these coordinates, the incident wave on the left boundary

$$
\Psi_{i}=e^{-i k_{a} x_{L}} e^{-i k_{a} t^{\prime}},
$$


where $x_{L}$ is the distance from the center of the cylinder to the left boundary. In our coordinates, the incident wave on the left boundary is:

$$
\Psi_{i}=e^{-i k_{a} t} .
$$

Therefore, the analytic solution must be modified as

$$
\begin{aligned}
\Psi^{-}(r, \phi, t)= & \sum_{n=0}^{\infty} i^{n} \varepsilon_{n} \cos (n \phi)\left[J_{n}\left(k_{a} r\right)-\frac{J_{n}\left(k_{a} a\right)}{H_{n}^{(1)}\left(k_{a} a\right)} H_{n}^{(1)}\left(k_{a} r\right)\right] \\
\Psi^{+}(r, \phi, t)= & e^{i k_{a} x_{L}} e^{-i k_{a} t},
\end{aligned}
$$

For $t=10$ periods, the analytic solution (taking the first ten terms in the series) for the perfect-conductor case is plotted in Fig. 9. Comparing Fig. 9 to Fig. 8, we find that the magnetic field distributions of the two solutions in air are in good agreement. The numerical solution also demonstrates a tendency of penetration of the wave into a good conductor. Deeper penetration occurs on the illuminated side than on the shadowed side. The difference between the two solutions near the artificial boundary comes from the fact that its distance from the center is not infinite. This trade-off, however, makes the numerical implementation on a computer possible and efficient.

In the low-frequency case, the waves in both regions have the following analytic forms:

$$
\begin{aligned}
\Psi^{-}(r, \phi, t)= & \sum_{n=0}^{\infty} i^{n} \varepsilon_{n} \cos (n \phi)\left[J_{n}\left(k_{a} r\right)+a_{n} H_{n}^{(1)}\left(k_{a} r\right)\right] \\
& e^{i k_{a} x_{L}} e^{-i k_{a} t} \\
\Psi^{+}(r, \phi, t)= & \sum_{n=0}^{\infty} i^{n} \varepsilon_{n} \cos (n \phi) b_{n} J_{n}(p r) e^{i k_{a} x_{L}} e^{-i k_{a} t}
\end{aligned}
$$

where $p \approx \sqrt{i \omega L^{2} \sigma_{m} \mu_{m}}$. Using interface conditions, the coefficients $a_{n}$ and $b_{n}$ are determined from the following equations:

$$
\begin{aligned}
J_{n}\left(k_{a} a\right)+a_{n} H_{n}^{(1)}\left(k_{a} a\right) & =\mu_{r} b_{n} J_{n}(p a), \\
k_{a}\left[J_{n}^{\prime}\left(k_{a} a\right)+a_{n}\left(H_{n}^{(1)}\right)^{\prime}\left(k_{a} a\right)\right] & =b_{n} p J_{n}^{\prime}(p a),
\end{aligned}
$$

for $n=0,1,2, \ldots$. The first three coefficients in these two equations are calculated to be:

$$
a_{0}=-1.027247 \times 10^{-2}-i 9.971206 \times 10^{-2}
$$




$$
\begin{aligned}
& a_{1}=-2.004432 \times 10^{-15}-i 2.852592 \times 10^{-14} \\
& a_{2}=-1.817832 \times 10^{-29}-i 1.288663 \times 10^{-28} \\
& b_{0}=-1.106546 \times 10^{-8}+i 8.582522 \times 10^{-9} \\
& b_{1}=2.314644 \times 10^{-14}+i 3.575007 \times 10^{-14} \\
& b_{2}=3.629113 \times 10^{-21}-i 2.260305 \times 10^{-21}
\end{aligned}
$$

Taking the first three terms in the series, we plot the analytic solution for the low frequency case in -Fig. 12. Comparing this to Fig. 11, we find that the numerical solution using the far-field condition has a large numeric error although its field pattern is similar to that of the analytic solution. The error comes from the position where the radiation condition is applied. This condition requires $k_{a} r$ to be large. But in our low-frequency experiment, $k_{a} r$ is very small $\left(O\left(10^{-6}\right)\right)$. As a result, the error appeares to be large. On the other hand, if we expand the computation domain to get a large $k_{a} r$, the grid size will become too large to be implemented on a computer. Therefore a boundary condition which is appropriate to the case of low $k_{a} r$ is needed to solve the problem.

Comparison of figures 10 and 12 shows that the numerical solution using the near-field condition matches the analytic solution very well in the exterior region. The root mean square error for the whole computation domain is calculated to be $1.772813 \times 10^{-3}$. Both solutions at the points with $r=4,20,40,60$, and $\theta=0, \pi / 4, \pi / 2,3 \pi / 4, \pi$ are listed on Table 1 . It shows that the fields on the illuminated and shadowed sides are nearly the same because of a strong diffraction for the low-frequency wave. The difference of the inside field between the two solutions is due to the fact that the numerical solution is in transient state while the analytic solution is at steady state.

Vector potential is related to the scalar function $\Psi$ by the following:

$$
\mathrm{A}=\mu \Psi \mathrm{k} \text {. }
$$

Using this relation, we obtain three-dimensional plots of vector potentials in various cases (see Fig. 15 through 18). In each plot, we see the magnitude of $\Psi$ versus $x$ and y axes. From these plots, we can observe the variation of $\Psi$ over the whole computational domain. Fig. 15 and Fig. 16 are the numerical and analytic solutions for the high-frequency case respectively. Fig. 17 and Fig. 18 are the numerical and analytic solutions for the low-frequency case respectively. We need these plots to check the numerical results near the interface boundary. According to the interface conditions, the fields are 
continuous on the boundary. Therefore $\Psi$ must change smoothly near the boundary. No sharp jumps are allowed. Usually this is a strict test for a numerical result. Fig. 15 and Fig. 17 show that our numerical results pass the test. The $\Psi$ values change smoothly and no jumps happen near the boundary.

To prove that our implementation for an interface problem is correct, we need obtain a steady-state solution for the low-frequency case. Also to capture an accurate eddy current phenomenon, the grid separation must be chosen smaller than the estimated skin depth. This skin depth is defined in the case of a plane scatterer of infinite depth as:

$$
\delta=\sqrt{\frac{1}{\pi f \mu_{m} \sigma_{m}}},
$$

where $\mathrm{f}$ is the frequency of the incident wave, $\mu_{m}$ and $\sigma_{m}$ are the permeability and conductivity of the scatterer respectively. In the computations that follow, we assume the conductor to be graphite with $\sigma_{m}=4 \times 10^{4} S / M$ (MKS units), $\mu_{m}=\mu_{a}$ and $\epsilon_{m}=\epsilon_{a}$. The frequency of the incident wave is 6000 $\mathrm{Hz}$. In this case, the estimated skin depth is $3.248737 \mathrm{~cm}$. The grid size is $25 \times 25$ with the diameter of the conductor occupying 21 grids. A grid separation of $.6544985 \mathrm{~cm}$ is chosen. Therefore the grid separation is about $1 / 5$ of the estimated skin depth. The numerical results were obtained after one period from the beginning. It took 20 minutes CPU time on a CRAYXMP-24. Fig. 19 and Fig. 20 show the results after one period of time. Comparing the corresponding analytic solutions in Fig. 21 and Fig. 22, the numerical results were found to be satisfactory. A clear example of eddy current phenomenon is demonstrated. Fig. 23 is the history at a point $\mathrm{P}(3,0)$ inside the conductor. A wave phenomenon is clearly observed in this figure. This justifies the comment we made previously. Both regions are governed by wave phenomena. Fig. 24 shows the numerical result for a quarter period. In this plot, a mesh of 61 grids by 61 grids, with the conductor's diameter occupying 41 grids, is used. A grid separation of $.3272493 \mathrm{~cm}$ is chosen. A conductor with $\sigma_{m}=10^{4} \mathrm{~S} / \mathrm{M}, \mu_{m}=\mu_{a}$ and $\epsilon_{m}=\epsilon_{a}$ is assumed. The frequency of the incident wave is also $6000 \mathrm{~Hz}$. At a quarter period, the incident magnetic field becomes identically zero. However, from Fig. 24, we find a weak magnetic field still exists in the exterior region. At the beginning, the field penetrates from the exterior region into the interior region. During a quarter period, the interior field is so much stronger than the exterior field and thus it penetrates back to the exterior region through the interface 
conditions. Therefore the maximum value of $\Psi$ occurs inside the conductor at this time. For a general transient signal, the problem needs to be solved in the time domain so that this phenomenon can be understood further. Our procedure can deal with such situations. Corresponding calculations will be reported elsewhere .

\section{Concluding Remarks}

From our investigation, the following conclusions are obtained:

1. Implementation of the interface problem in this paper is proved to be satisfactory for both high- and low-frequency cases, by numerical experiments.

2. Implementation of the radiation condition is found effective in handling corner problems.

3. To solve the low-frequency eddy current problem, a boundary condition which is appropriate to the case of low $k_{a} r$ is needed. The boundary condition obtained has been shown to work very well in numerical experiments.

4. To obtain an accurate eddy current representation, the grid separation near the interface should be smaller than the estimated skin depth in (78).

5. In the low-frequency eddy current problem, our finite difference method calls for extremely fine time steps. As a result for good conductors such as aluminum, calculation of penetration of the field takes relatively larger time than that for conductors such as graphite. Thus an acceleration scheme is preferred so that calculations can be performed inexpensively. 


\section{A Appendix: Uniqueness of the Solution}

The problem considered can be written in the following form:

$$
\begin{aligned}
& \Psi_{t t}^{s}=\Delta \Psi^{s} \quad \text { in } \Omega_{A}, \\
& k_{m}^{2} \Psi_{t t}+l_{m}^{2} \Psi_{t}=\Delta \Psi \quad \text { in } \Omega, \\
& \Psi^{s}+\Psi^{i}=\Psi \quad \text { on } \Gamma, \\
& \frac{\partial \Psi^{s}}{\partial n}+\frac{\partial \Psi^{i}}{\partial n}=\frac{\partial \Psi}{\partial n} \quad \text { on } \Gamma, \\
& \Psi^{s}(x, y, 0), \Psi_{t}^{s}(x, y, 0)=0 \quad \text { in } \Omega_{A} \text {, } \\
& \Psi(x, y, 0), \Psi_{t}(x, y, 0)=0 \quad \text { in } \Omega \text {, } \\
& \Psi_{r}^{s}+\Psi_{t}^{s}+A(r) \Psi^{s}=0 \quad \text { on } \Gamma_{e} .
\end{aligned}
$$

Here $\Delta$ is the Laplacian operator. Subscripts ' $t$ ', ' $t t$ ', and ' $r$ ' represent partial derivatives. Superscripts ' $\mathrm{s}$ ' and ' $\mathrm{i}$ ' represent scattered wave and incident wave respectively. $\Gamma_{e}$ is the radiation boundary as shown in Fig. 25. Here we assume $\Gamma_{e}$ to be a circle. $A(r)=1 / 2 r$ for the high-frequency case and

$$
A(r)=\frac{1}{r}\left[-\frac{1}{\gamma-\ln 2+\ln k_{a}+\ln r}\right]
$$

for the low-frequency situation.

First, assume that the problem has two solutions $u_{1}(x, y, t)$ and $u_{2}(x, y, t)$ and $v(x, y, t)=u_{2}(x, y, t)-u_{1}(x, y, t)$. Then

$$
\begin{array}{rlr}
v_{t t} & =\Delta v & \text { in } \Omega_{A}, \\
k_{m}^{2} v_{t t}+l_{m}^{2} v_{t} & =\Delta v & \text { in } \Omega, \\
v^{-} & =v^{+} & \text {on } \Gamma, \\
\frac{\partial v^{-}}{\partial n} & =\frac{\partial v^{+}}{\partial n} & \text { on } \Gamma, \\
v(x, y, 0), v_{t}(x, y, 0) & =0 & \text { in } \Omega_{A}, \Omega, \\
v_{r}+v_{t}+A(r) v & =0 & \text { on } \Gamma_{e} .
\end{array}
$$

We construct a function

$$
E(t)=\frac{1}{2} \iint_{\Omega_{A}}\left(v_{t}^{2}+\nabla v \cdot \nabla v\right) d \Omega_{A}+\frac{1}{2} \iint_{\Omega}\left(k_{m}^{2} v_{t}^{2}+\nabla v \cdot \nabla v\right) d \Omega,
$$

where $\nabla$ is the gradient operator and - is the operator for the inner product. Taking the derivative of $\mathrm{E}(\mathrm{t})$ with respect to $t$, we obtain 


$$
\begin{aligned}
E^{\prime}(t) & =\iint_{\Omega_{A}}\left(v_{t} v_{t t}+\nabla v \cdot \nabla v_{t}\right) d \Omega_{A}+\iint_{\Omega}\left(k_{m}^{2} v_{t} v_{t t}+\nabla v \cdot \nabla v_{t}\right) d \Omega \\
& =\iint_{\Omega_{A}}\left(v_{t} \Delta v+\nabla v \cdot \nabla v_{t}\right) d \Omega_{A}+\iint_{\Omega}\left(v_{t} \Delta v+\nabla v \cdot \nabla v_{t}-l_{m}^{2} v_{t}^{2}\right) d \Omega \\
& =\iint_{\Omega_{A}}\left(\nabla \cdot v_{t} \nabla v\right) d \Omega_{A}+\iint_{\Omega}\left(\nabla \cdot v_{t} \nabla v-l_{m}^{2} v_{t}^{2}\right) d \Omega \\
& =\int_{S_{A}} v_{t} \nabla v \cdot \underline{n} d s+\int_{\Gamma} v_{t} \nabla v \cdot \underline{n} d s-\iint_{\Omega} l_{m}^{2} v_{t}^{2} d \Omega \\
& =\int_{\Gamma_{e}} v_{t} v_{n} d s-\int v_{t} v_{n} d s+\int_{\Gamma} v_{t} v_{n} d s-\iint_{\Omega} l_{m}^{2} v_{t}^{2} d \Omega \\
& =\int_{\Gamma_{e}} v_{t} v_{n} d s-\iint_{\Omega} l_{m}^{2} v_{t}^{2} d \Omega \\
& =\int_{\Gamma_{e}} v_{t} v_{r} d s-\iint_{\Omega} l_{m}^{2} v_{t}^{2} d \Omega \\
& =\int_{\Gamma_{e}} v_{t}\left(-v_{t}-A(r) v\right) d s-\iint_{\Omega} l_{m}^{2} v_{t}^{2} d \Omega
\end{aligned}
$$

Thus we have

$$
E^{\prime}(t)+\int_{\Gamma_{e}} A(r) v v_{t} d s=-\int_{\Gamma_{e}} v_{t}^{2} d s-\iint_{\Omega} l_{m}^{2} v_{t}^{2} d \Omega
$$

The right side of the equation above is less than or equal to zero. Then

$$
E^{\prime}(t)+\frac{\partial}{\partial t} \int_{\Gamma_{e}} \frac{1}{2} A(r) v^{2} d s \leq 0
$$

From the initial condition, $v(x, y, 0)=0, v_{t}(x, y, 0)=0$, and $E(0)=0$. It follows that

$$
E(t)+\int_{\Gamma_{e}} \frac{1}{2} A(r) v^{2} d s \leq 0
$$

so that

$$
E(t) \leq-\int_{\Gamma_{e}} \frac{1}{2} A(r) v^{2} d s
$$

From the structure of $\mathrm{A}(\mathrm{r})$ (section 4 ) for both high- and low-frequency waves, $A(r)>0$ on $\Gamma_{e}$. As a result, $E(t) \leq 0$. But from the definition, $E(t) \geq 0$. Thus the possibility is $E(t)=0$. This leads to $v_{t}(x, y, t)=0$, i.e., $\mathrm{v}$ is constant for the time. Since $v(x, y, 0)=0, v(x, y, t)=0$ for all time and all space. This means $u_{1}(x, y, t)=u_{2}(x, y, t)$. Therefore we conclude that the problem has a unique solution. 


\section{References}

[1] S. I. Hariharan and R. C. MacCamy. Integral Equation Problems for Eddy Current Problems. J. Comput. Phys., Vol. 45, No. 1, 1982.

[2] S. I. Hariharan and R. C. MacCamy. Low Frequency Acoustic and Electromagnetic Scattering. ICASE Report No. 83-43, 1983.

[3] R. C. MacCamy and E. Stephan. Solution Procedures for ThreeDimensional Eddy Current Problem. J. of Math. Anal. and its Appli., Vol. 101, No. 2, 1984.

[4] G. Kriegsmann and C. S. Morawetz. Numerical Solutions of Exterior Problems with Reduced Wave Equation. J. Comp. Phys., Vol. 28, 1978.

[5] G. Kriegsmann and C. S. Morawetz. Solving the Helmholtz Equation for Exterior Problems with a Variable Index of Refraction. SIAM J. Sci. Stat. Comput., Vol. 1, 1980.

[6] A. Taflove and K. R. Umashankar. Solution of Complex Electromagnetic Penetration and Scattering Problems in Unbounded Regions in Computational Methods for Infinite Domain Media-Structure Interaction. A. J. Dalinowski, ed., 1981.

[7] S. I. Hariharan and T. H. Moulden. Numerical Methods for Partial Differential Equations. Longmans Scientific and Technical, 1986.

[8] B. Engquist and A. Majda. Radiation Boundary Conditions for Acoustic and Elastic Wave Calculations. Comm. Pure Appl. Math., Vol. 32, 1979.

[9] J. Van Bladel. Electromagnetic Fields. Hemisphere Publishing Corporation, 1985.

[10] R. F. Harrington. Electromagnetic Fields. McGraw-Hill Book Company, 1961.

[11] S. I. Hariharan and S. I. Sudharsanan. Wave Envelope Technique for Multimode Waveguide Problems. J. of Sc. Computing, Vol. 2, No. 4, 1987.

[12] Jin Au Kong. Electromagnetic Wave Theory. John Wiley \& Sons, 1986 
[13] R. C. MacCamy and M. Suri. A Time-Dependent Interface Problem for Two-Dimensional Eddy Currents. Quart. App. Math., Vol. XLIV, No. $4,1987$.

Table 1. Solution Comparison for Low Frequency

\begin{tabular}{|c|c|c|c|}
\hline Radius & Grid & Numerical & \multicolumn{1}{c|}{ Analytic } \\
\hline \multirow{6}{*}{20} & $(80,0)$ & $0.8035427 \mathrm{D}-01$ & $0.7879715 \mathrm{D}-01$ \\
& $(74,14)$ & $0.7969991 \mathrm{D}-01$ & $0.7815606 \mathrm{D}-01$ \\
& $(60,20)$ & $0.8028407 \mathrm{D}-01$ & $0.7879725 \mathrm{D}-01$ \\
& $(45,14)$ & $0.8197310 \mathrm{D}-01$ & $0.8042111 \mathrm{D}-01$ \\
& $(40,0)$ & $0.8021339 \mathrm{D}-01$ & $0.7879735 \mathrm{D}-01$ \\
\hline \multirow{6}{*}{40} & $(100,0)$ & $0.1246082 \mathrm{D}+00$ & $0.1227901 \mathrm{D}+00$ \\
& $(88,28)$ & $0.1239659 \mathrm{D}+00$ & $0.1221490 \mathrm{D}+00$ \\
& $(60,40)$ & $0.1245336 \mathrm{D}+00$ & $0.1227901 \mathrm{D}+00$ \\
& $(31,28)$ & $0.1250927 \mathrm{D}+00$ & $0.1232822 \mathrm{D}+00$ \\
& $(20,40)$ & $0.1244591 \mathrm{D}+00$ & $0.1227902 \mathrm{D}+00$ \\
\hline \multirow{6}{*}{60} & $(120,0)$ & $0.1503752 \mathrm{D}+00$ & $0.1485242 \mathrm{D}+00$ \\
& $(102,42)$ & $0.1498472 \mathrm{D}+00$ & $0.1478831 \mathrm{D}+00$ \\
& $(60,60)$ & $0.1503764 \mathrm{D}+00$ & $0.1485243 \mathrm{D}+00$ \\
& $(17,42)$ & $0.1505956 \mathrm{D}+00$ & $0.1486387 \mathrm{D}+00$ \\
& $(0,20)$ & $0.1503784 \mathrm{D}+00$ & $0.1485243 \mathrm{D}+00$ \\
\hline \multirow{6}{*}{4} & $(64,0)$ & $0.1710767 \mathrm{D}-13$ & $0.2201674 \mathrm{D}-04$ \\
& $(62,2)$ & $0.1975745 \mathrm{D}-18$ & $-0.1127474 \mathrm{D}-05$ \\
& $(60,4)$ & $0.1719809 \mathrm{D}-13$ & $0.2201422 \mathrm{D}-04$ \\
& $(57,2)$ & $0.3046863 \mathrm{D}-14$ & $0.1069381 \mathrm{D}-04$ \\
& $(56,0)$ & $0.1731365 \mathrm{D}-13$ & $0.2201171 \mathrm{D}-04$ \\
\hline
\end{tabular}

*In Double Precision expression. 


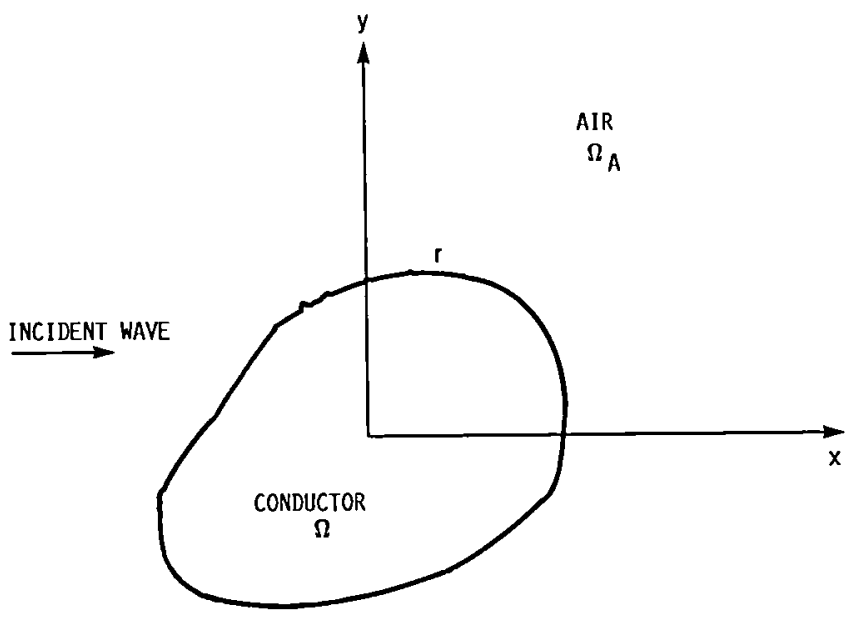

FIgURE 1. - TYPICAL SITUATION OF THE PROBLEM.

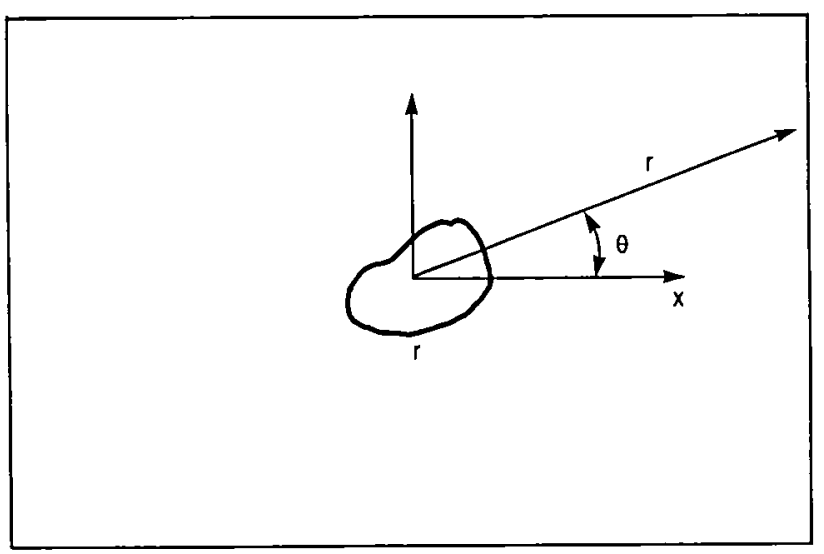

FIGURE 2. - COORDINATES OF THE COMPUTATIONAL DOMAIN.

F.D.: FORWARD DIFFERENCE

B.D.: BACKWARD DIFFERENCE

N. : NO EFFECT

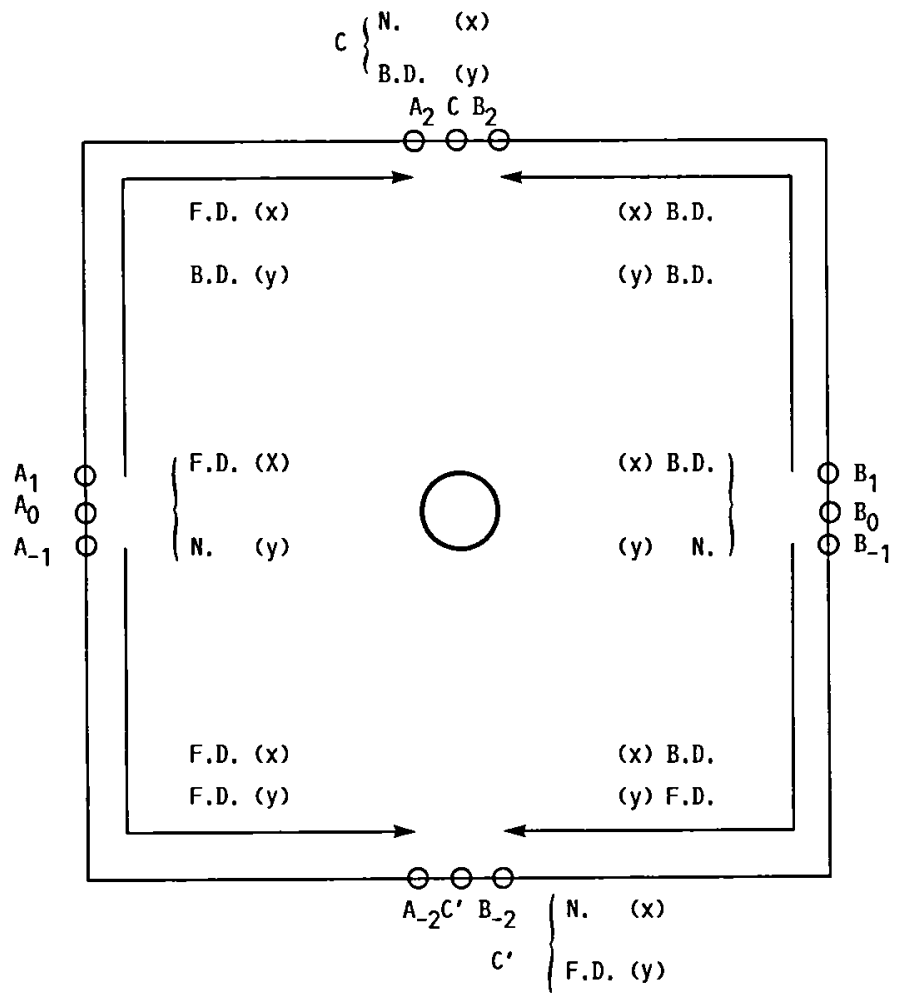

FIGURE 3. - IMPLEMENTATION OF THE RADIATION CONDITION.

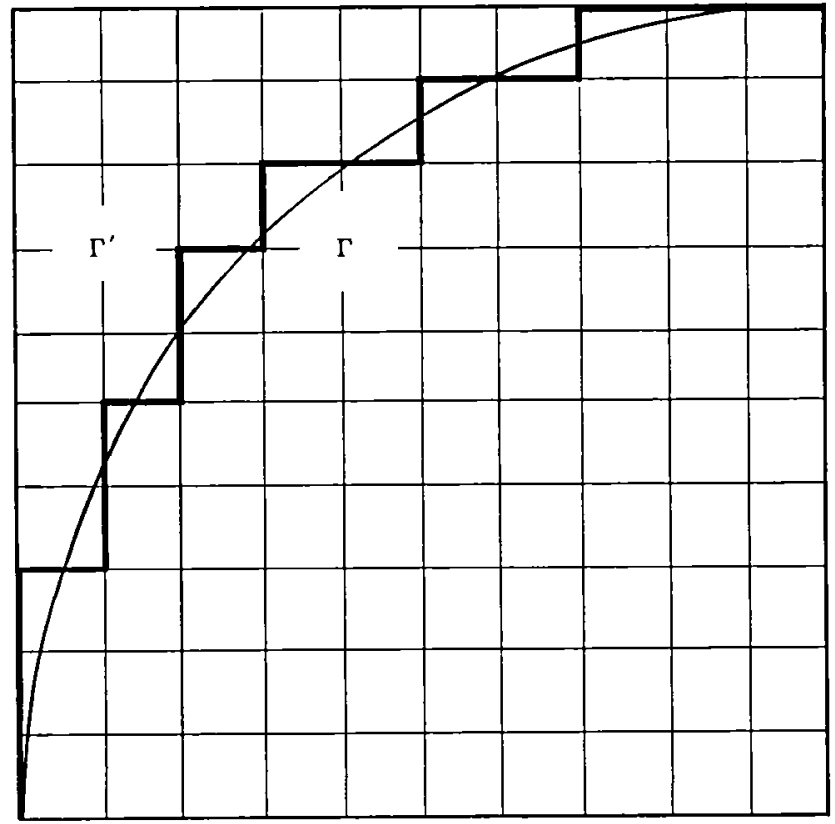

FIGURE 4. - APPROXIMATING A CURVE $\Gamma$ WITH A POLYGON $\Gamma$ : 


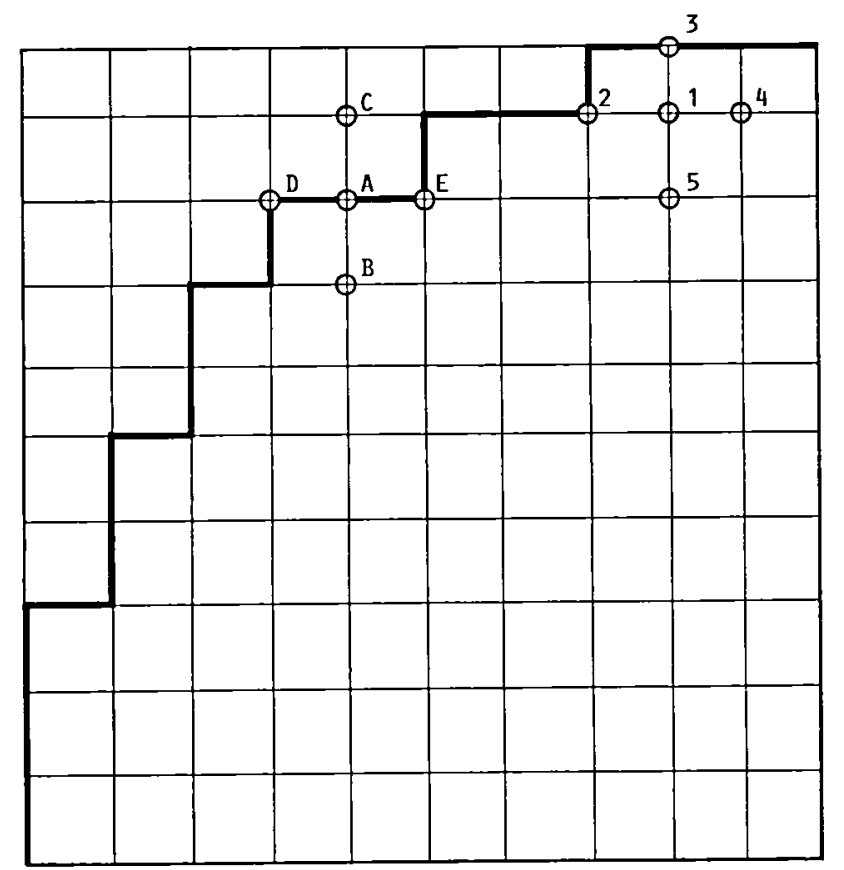

FIGURE 5. - GRID POINTS NEAR THE INTERFACE BOUNDARY WHEN $\mu_{\mathrm{r}} \neq 1$.

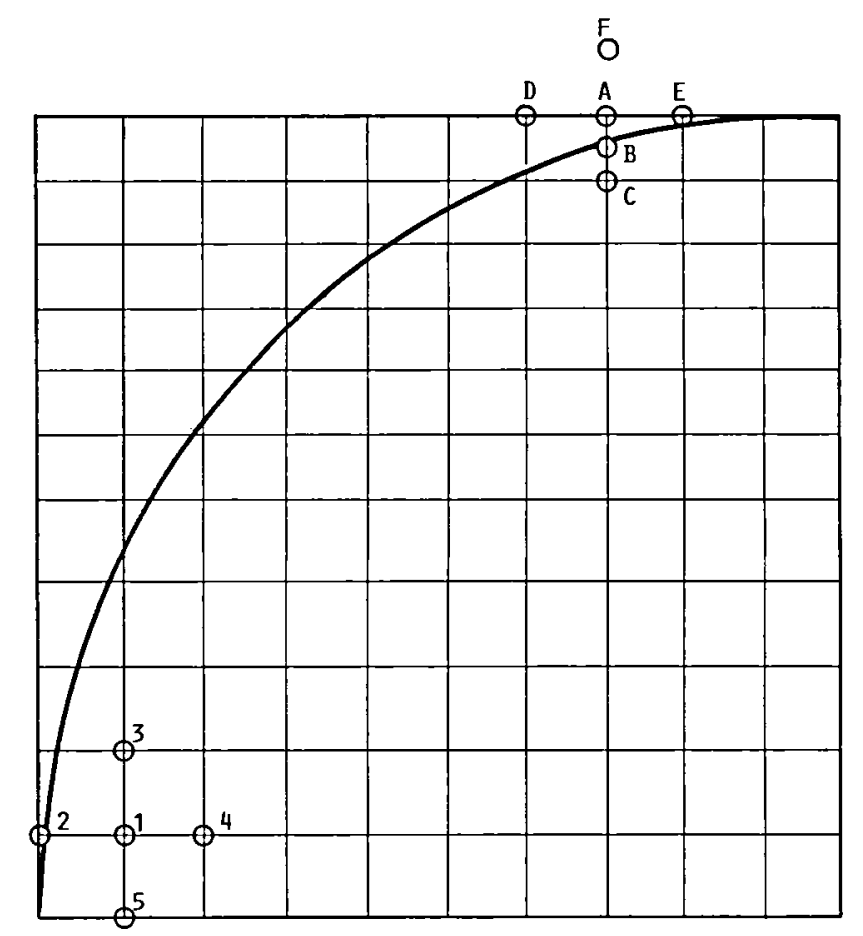

FIGURE 6. - GRID POINTS NEAR THE INTERFACE BOUNDARY WHEN $\mu_{r}=1$.

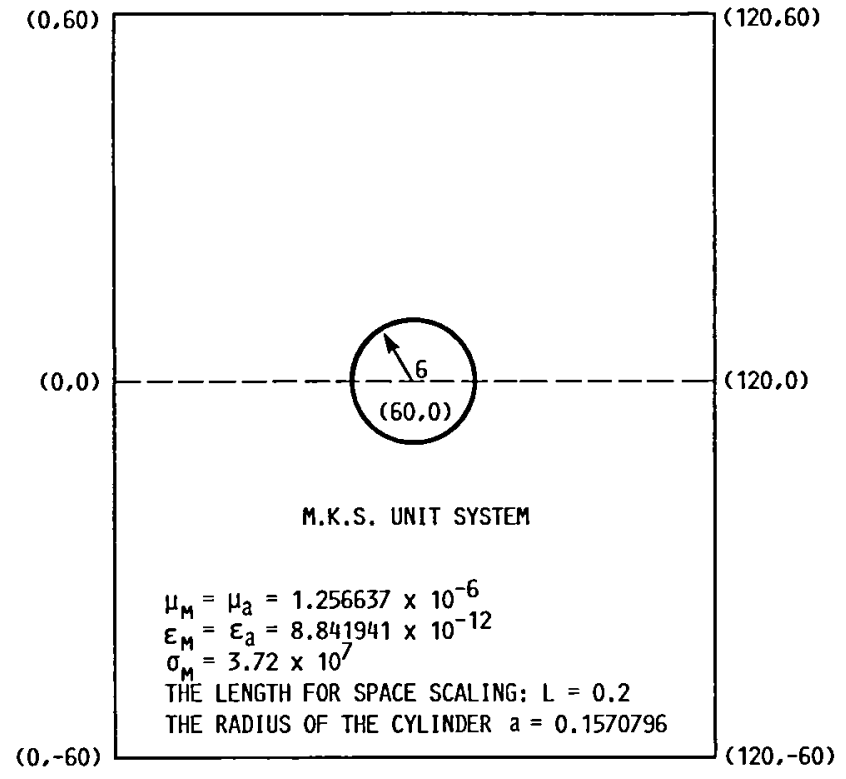

FIGURE 7. - GRID SIZE FOR THE NUMERICAL EXPERIMENT IN SECTION 6.

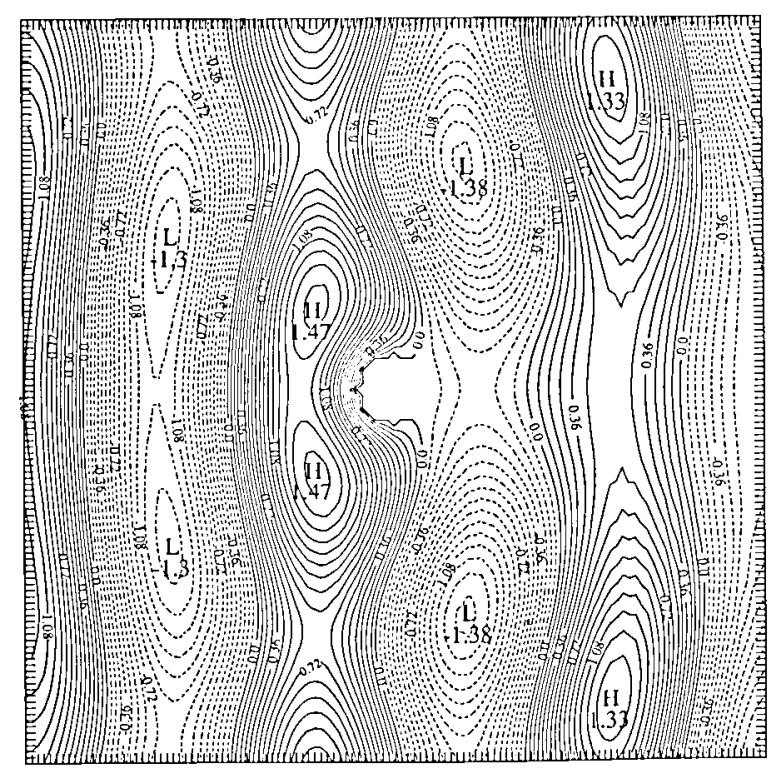

MAGNETIC FIELD LINE, NUMERICAL

FIGURE 8. - CONTOUR PLOT OF THE NUMERICAL SOLUTION FOR THE HIGH-FREQUENCY CASE. $f=2.387324 \times 10^{\circ} \mathrm{Hz}$ : GRID SIZE AND OTHER PARAMETERS ARE SHOWN IN FIG. 7; RESULT WAS OBTAINED AFTER 10 PERIODS FROM THE BEGINNING. 


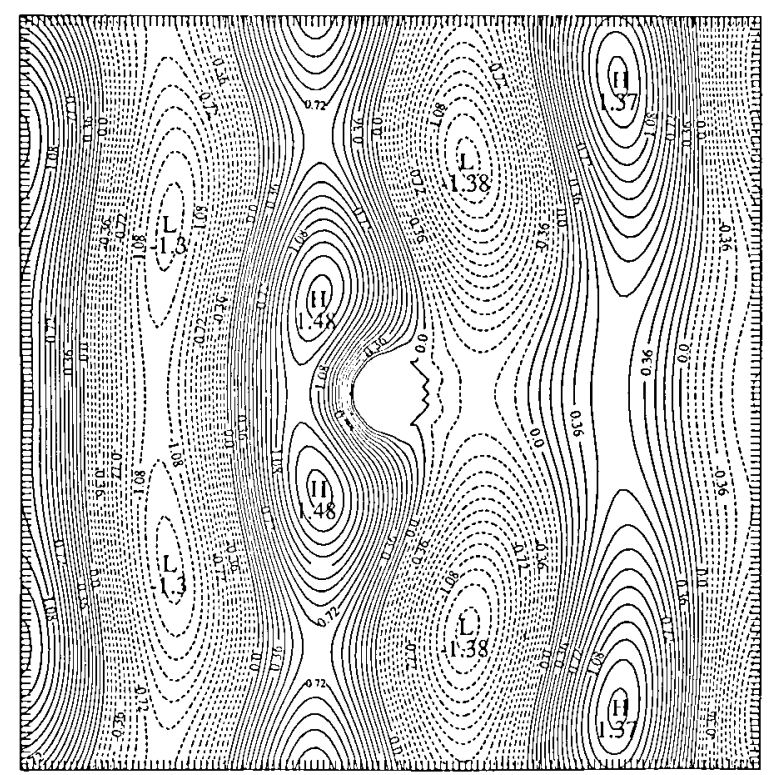

MAGNETIC FIELD LINE, ANALYTIC

FIGURE 9. - CONTOUR PLOT OF THE CORRESPONDING ANALYTIC SOLUTION OF FIG. 8.

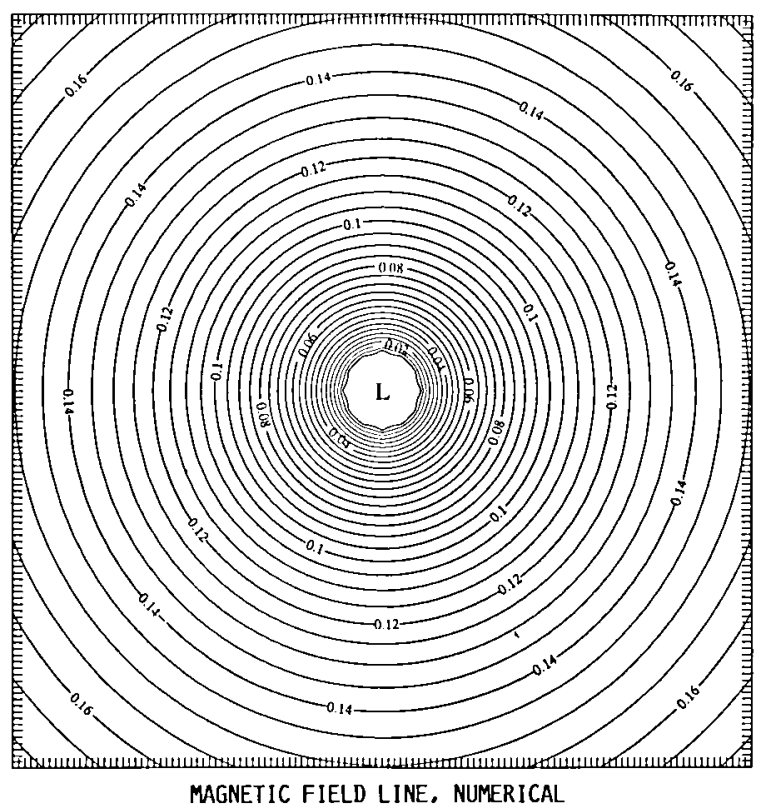

FIGURE 10. - CONTOUR PLOT OF THE NUMERICAL SOLUTION, USING THE NEAR-FIELD BOUNDARY CONDIUION, FOR THE LOW-FREQUENCY

CASE. $f=60 \mathrm{~Hz}$ : THE GRID SIZE AND OTHER PARAMETERS ARE SHOWN IN FIG. 7 ; THE RESULT WAS OBTAINED AFTER $2.513274 \times$ $10^{-4}$ PERIOD FROM THE BEGINNING.

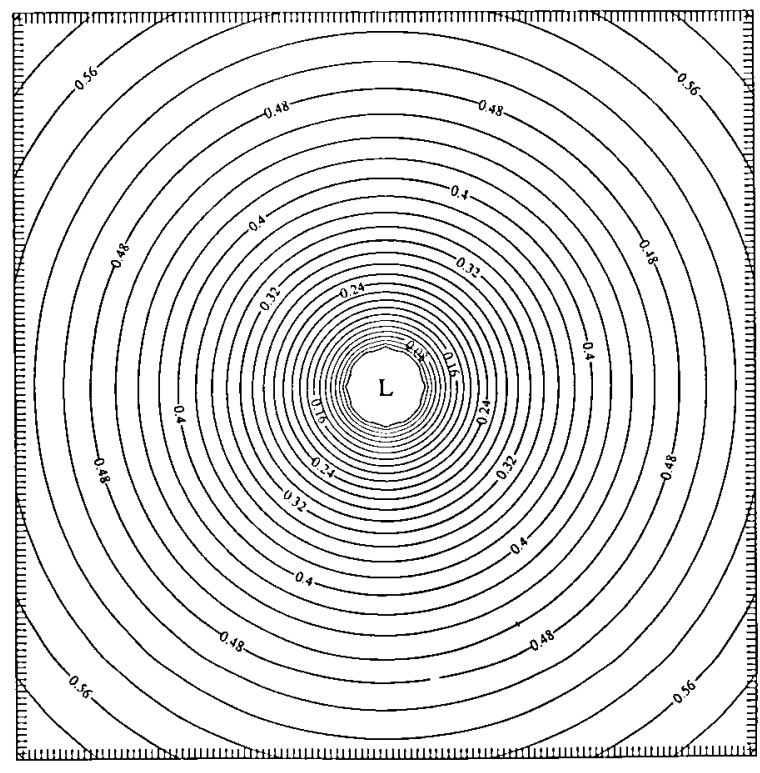

MAGNETIC FIELD LINE, NUMERICAL

FIGURE 11. - CONTOUR PLOT OF THE NUMERICAL SOLUTION, USING THE FAR-FIELD BOUNDARY CONDITTION, FOR THE LOW-FREQUENCY CASE. $f=60 \mathrm{~Hz}$; GRID SIZE AND OTHER PARAMETERS ARE SHOWN IN FIG. 7: RESULT WAS OBTAINED AFTER $2.513274 \times 10^{-4}$ PERIOD FROM THE BEGINNING.

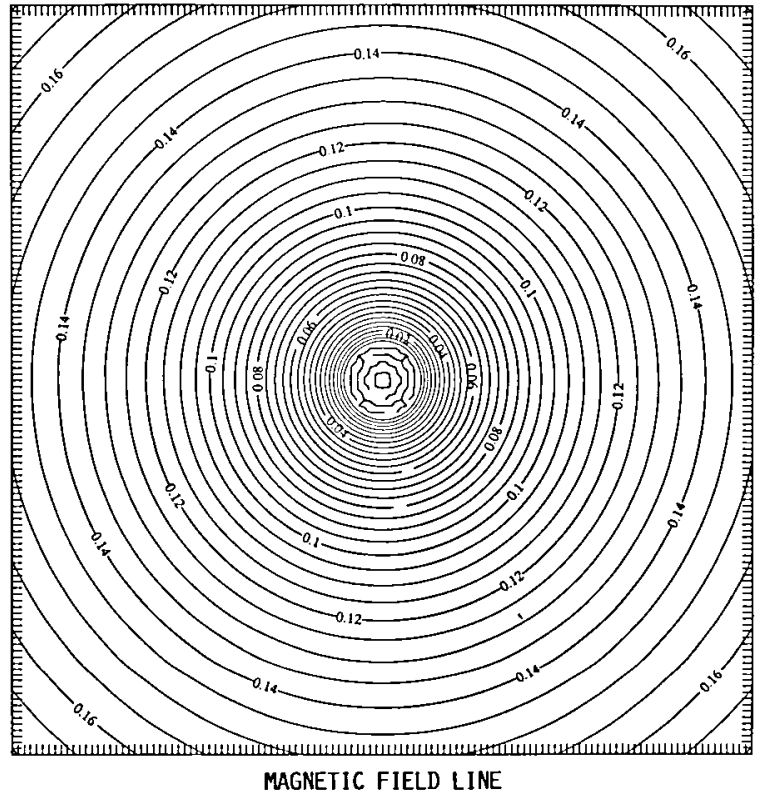

FIGURE 12. - CONTOUR PLOT OF THE CORRESPONDING ANALYTIC SOLUTION OF FIG. 10. 

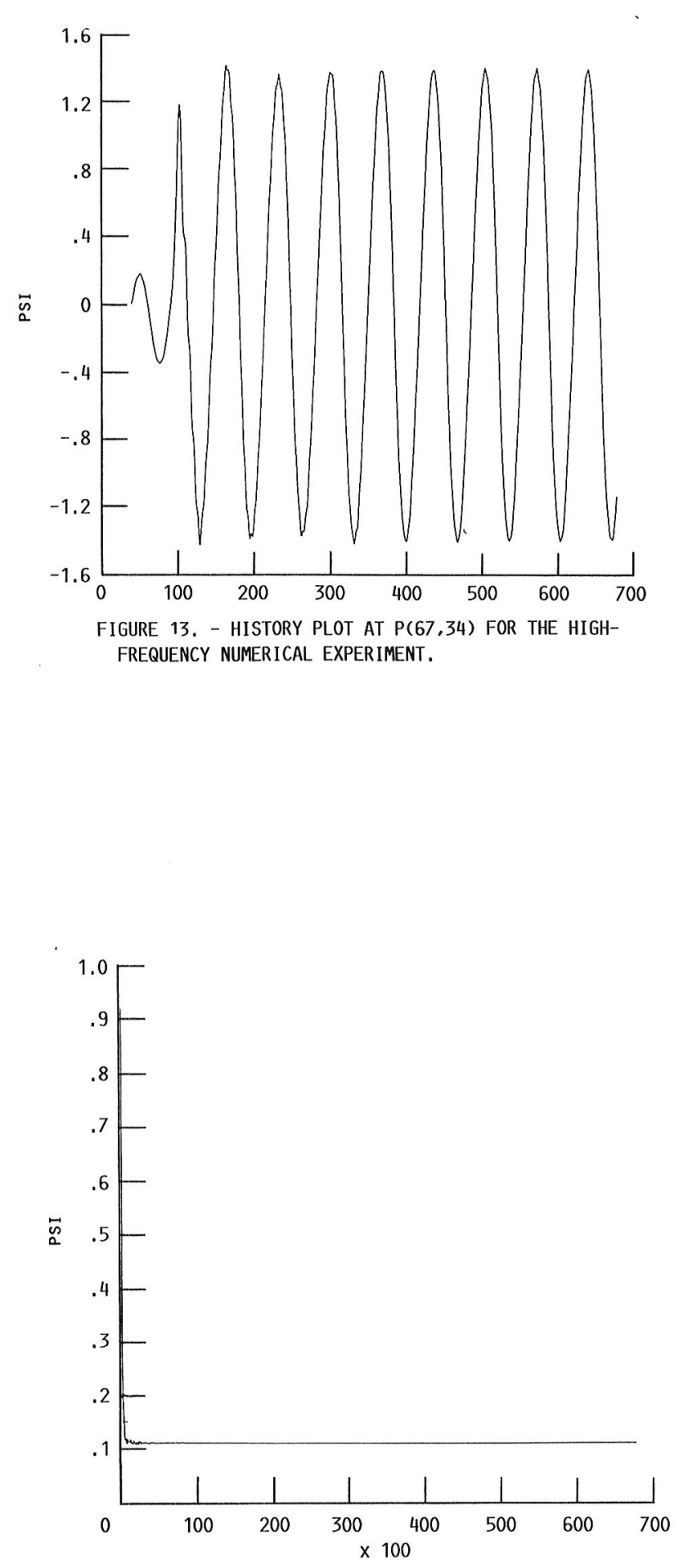

FIGURE 14. - CONCLUDED.

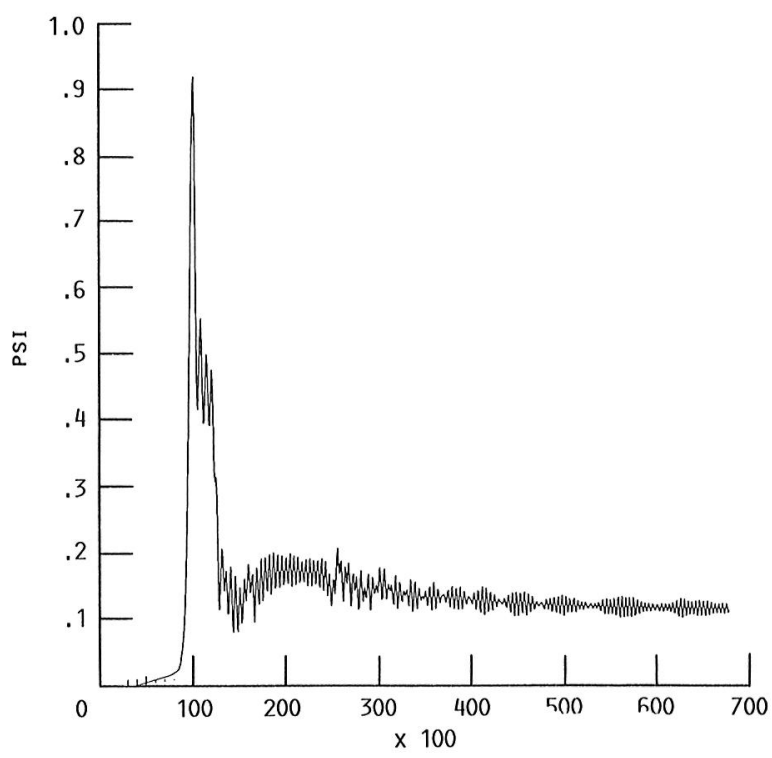

FIGURE 14. - HISTORY PLOT AT P $(67,34)$ FOR THE $60 \mathrm{~Hz}$ NUMERICAL EXPERIMENT.

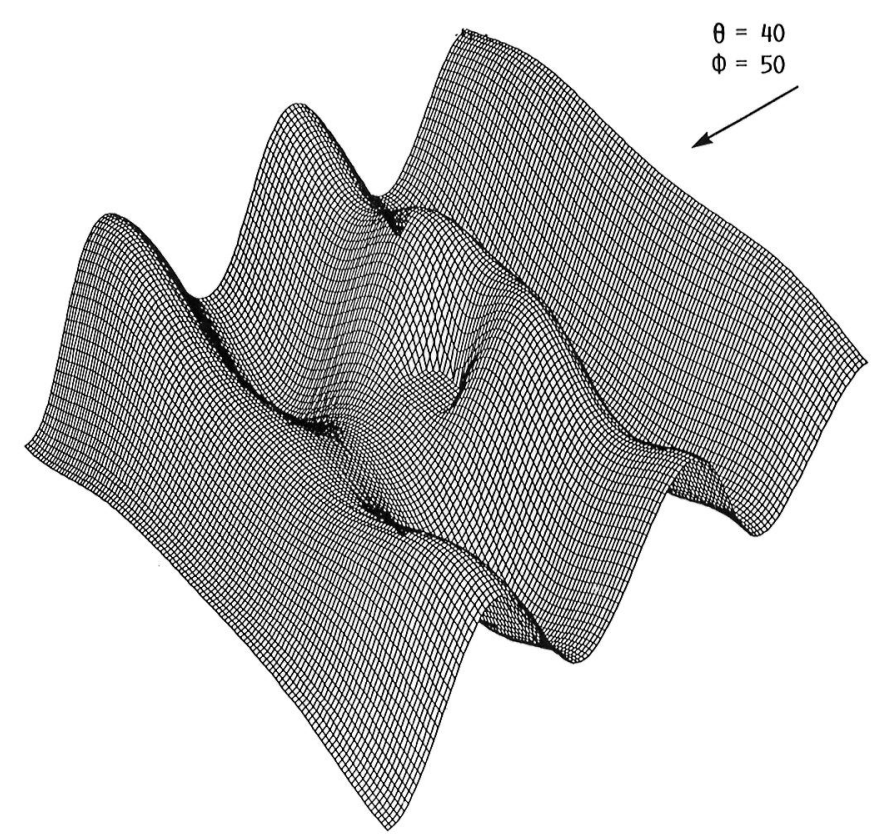

VECTOR POTENTIAL NUMERICAL

FIGURE 15. - SURFACE PLOT OF THE NUMERICAL SOLUTION FOR THE HIGHFREQUENCY CASE. GRID SIZE AND PARAMETERS ARE THE SAME AS THOSE IN FIG. 8. 


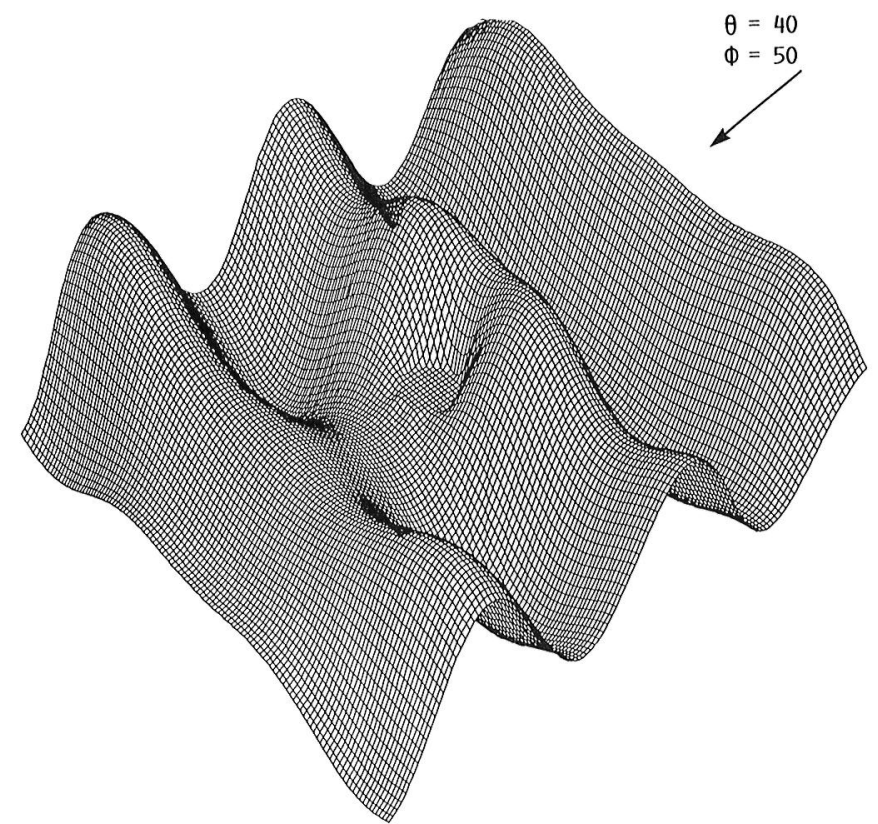

VECTOR POTENTIAL, ANALYTIC

FIGURE 16. - SURFACE PLOT OF THE CORRESPONDING ANALYTIC SOLUTION OF FIG. 15.

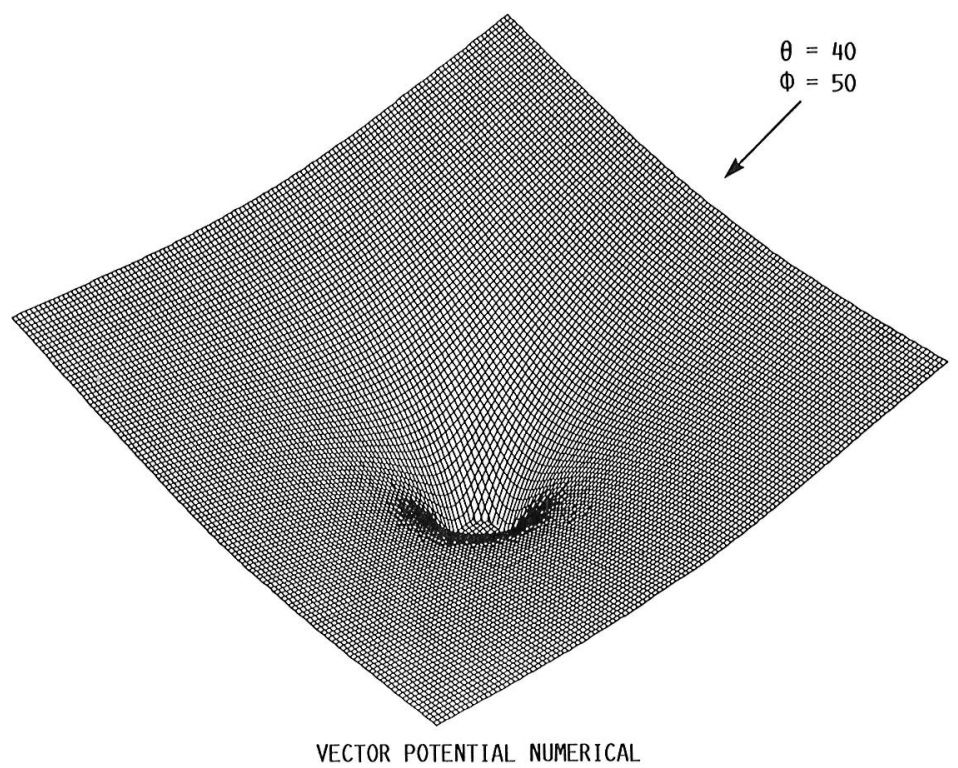

FIGURE 17. - SURFACE PLOT OF THE NUMERICAL SOLUTION FOR THE LOW-FREQUENCY CASE. GRID SIZE AND PARAMETERS ARE THE SAME AS THOSE IN FIG. 10. 


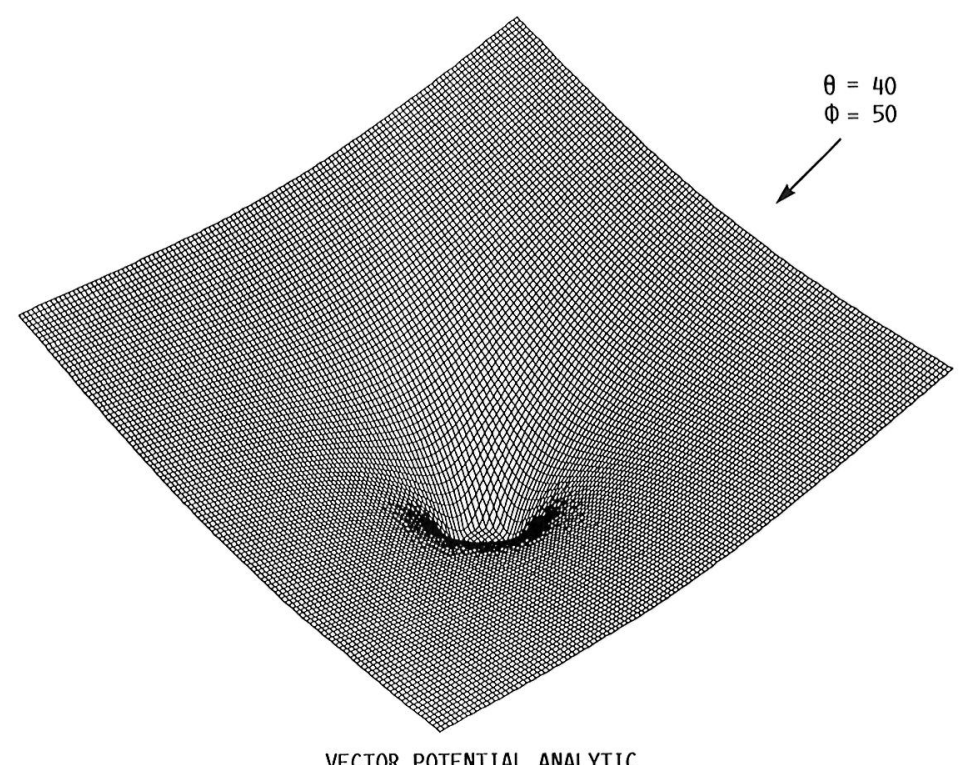

VECTOR POTENTIAL ANALYTIC

FIGURE 18. - SURFACE PLOT OF THE CORRESPONDING ANALYTIC SOLUTION OF FIG. 17.

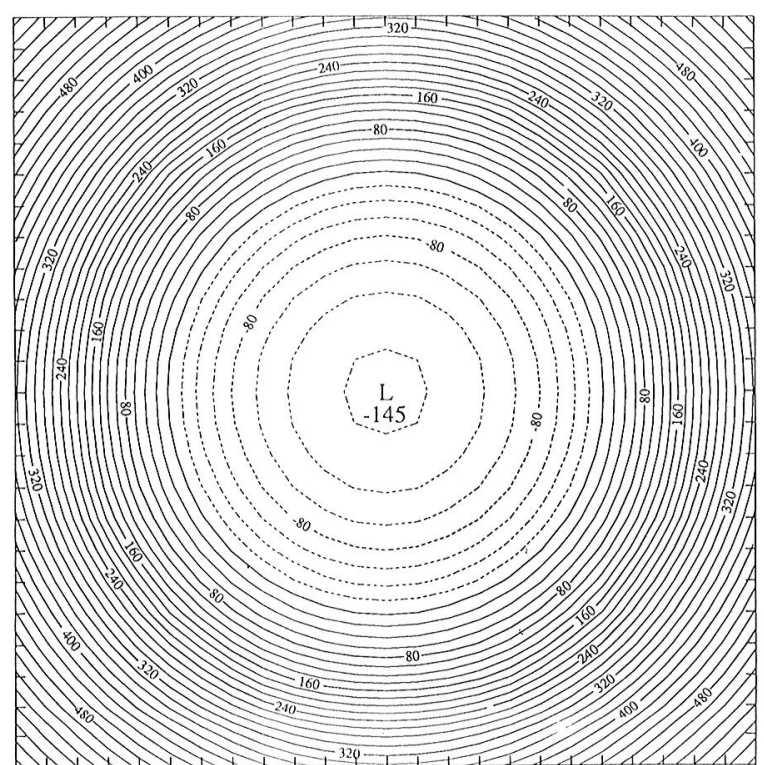

MAGNETIC FIELD LINE, NUMERICAL

FIGURE 19. - CONTOUR PLOT OF THE NUMERICAL RESULT IN SECTION 7. $f=6000 \mathrm{~Hz} ; \sigma_{M}=4.0 \times 10^{4} \mathrm{~S} / \mathrm{M} ; \varepsilon_{\mathrm{M}}=\varepsilon_{\mathrm{a}}$; $\mu_{M}=\mu_{a} ;$ GRID SIZE IS $25 \times 25$ WITH THE DIAMETER OF THE CONDUCTOR OCCUPYING 21 GRIDS; THE GRID SEPARATION IS $.6544985 \mathrm{cM}$; THE RESULT WAS OBTAINED AFTER ONE PERIOD FROM THE BEGINNING.

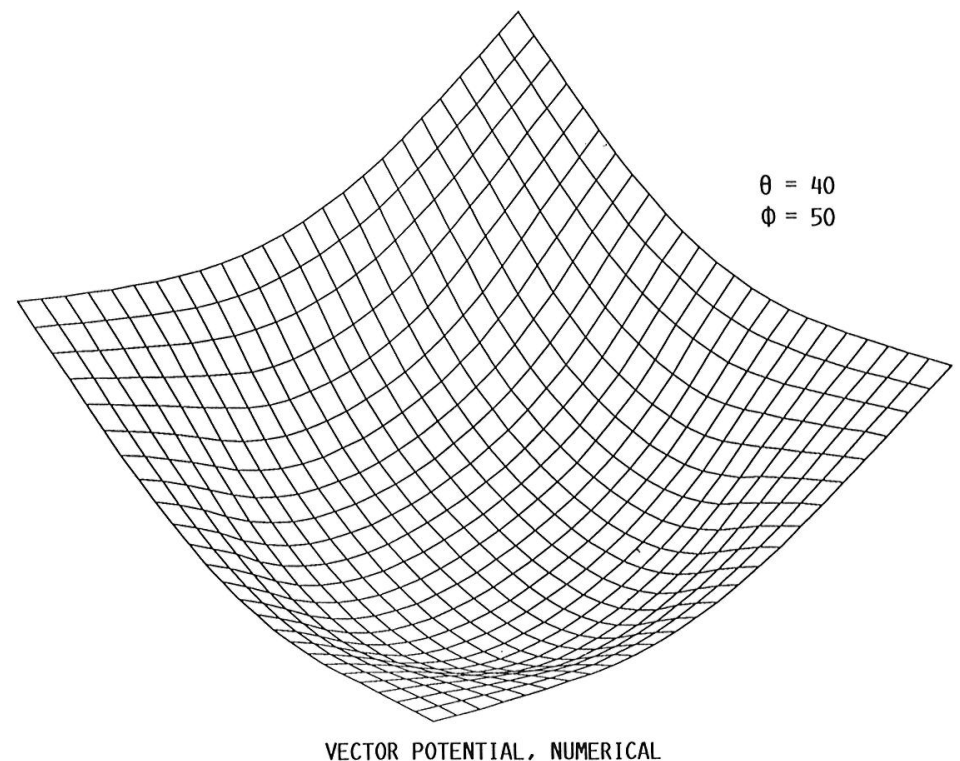

FIGURE 20. - SURFACE PLOT OF THE RESULT IN FIG. 19. 


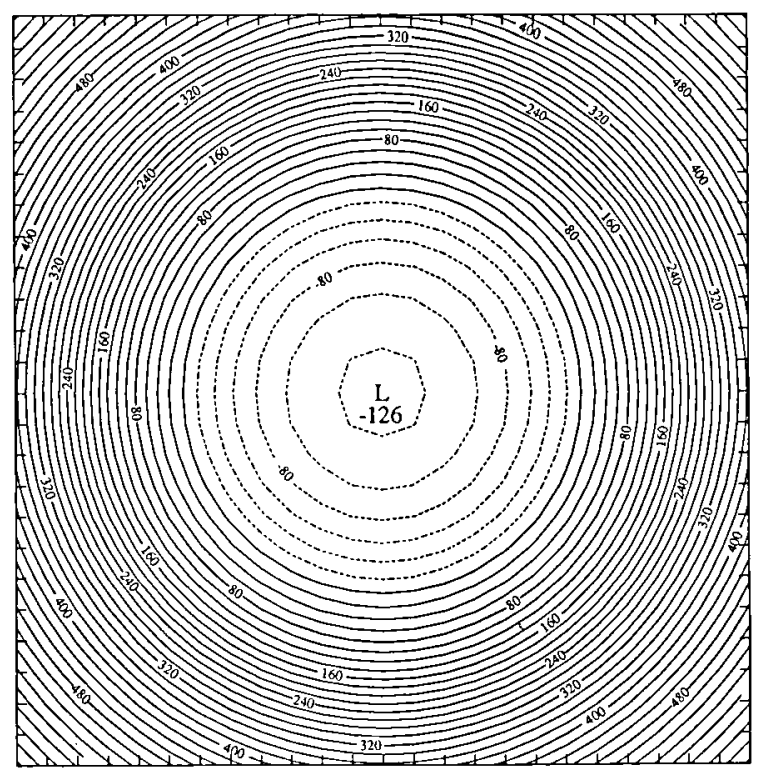

MAGNETIC FIELD LINE, ANALYTIC

FIGURE 21. - CONTOUR PLOT OF THE CORRESPONDING ANALYTIC SOLUTION OF FIG. 19.

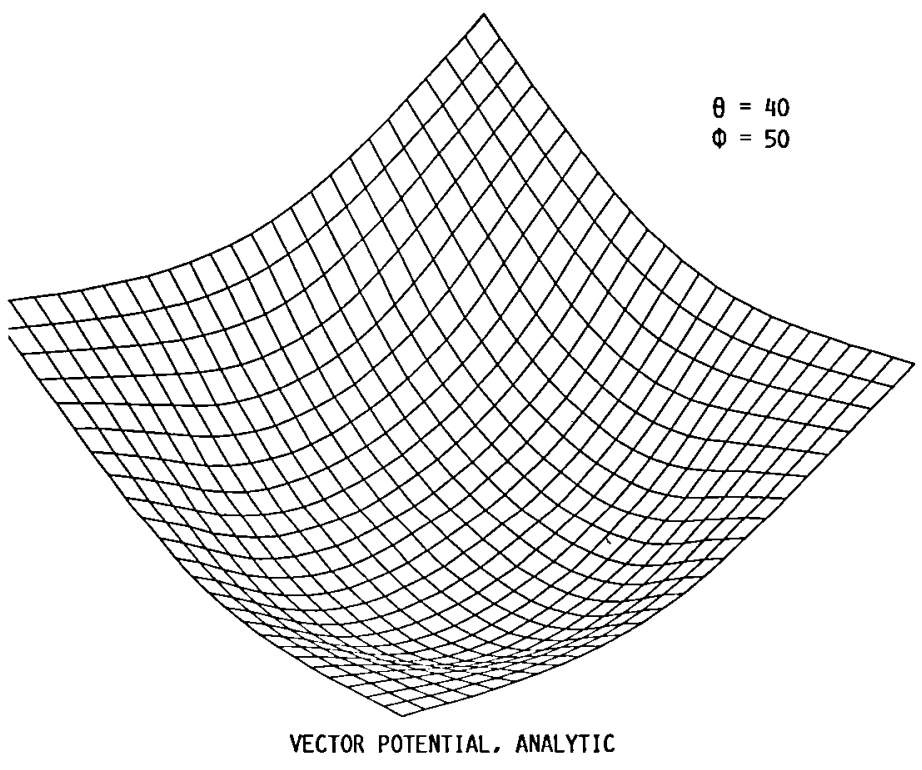

FIGURE 22. - SURFACE PLOT OF THE RESULT IN FIG. 21.

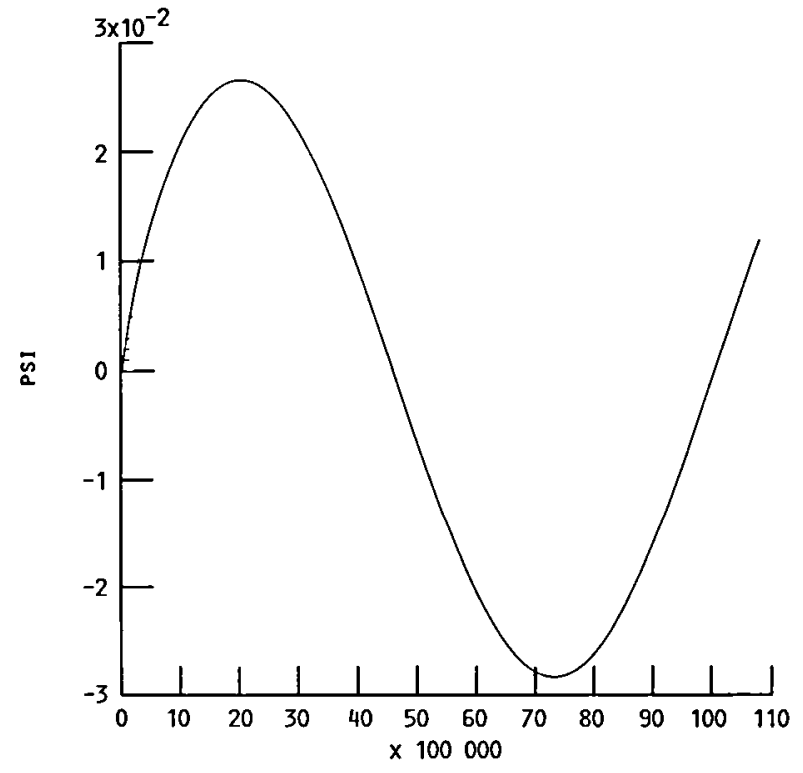

FIGURE 23. - HISTORY PLOT AT P(3.0) WHICH IS INSIDE THE CONDUCTOR, FOR THE RESULT IN FIG. 19. 


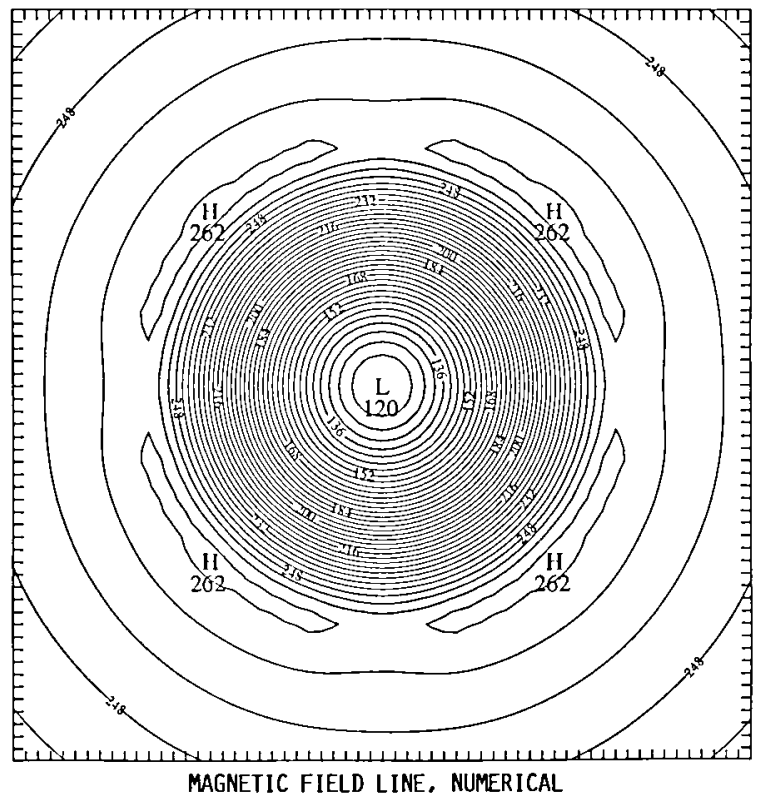

FIGURE 24.- CONTOUR PLOT OF THE NUMERICAL SOLUTION AT A QUARTER PERIOD. $f=6000 \mathrm{~Hz} ; \sigma_{M}=10^{+4} \mathrm{~S} / \mathrm{M} ; \mu_{M}=\mu_{\mathrm{a}}$ : $\varepsilon_{M}=\varepsilon_{\mathrm{a}}$ : GRID SIZE IS $61 \times 61$ WITH THE DIAMETER OF THE CONDUCTOR OCCUPYING 41 GRIDS; GRID SEPARATION IS $.3272493 \mathrm{~cm}$.

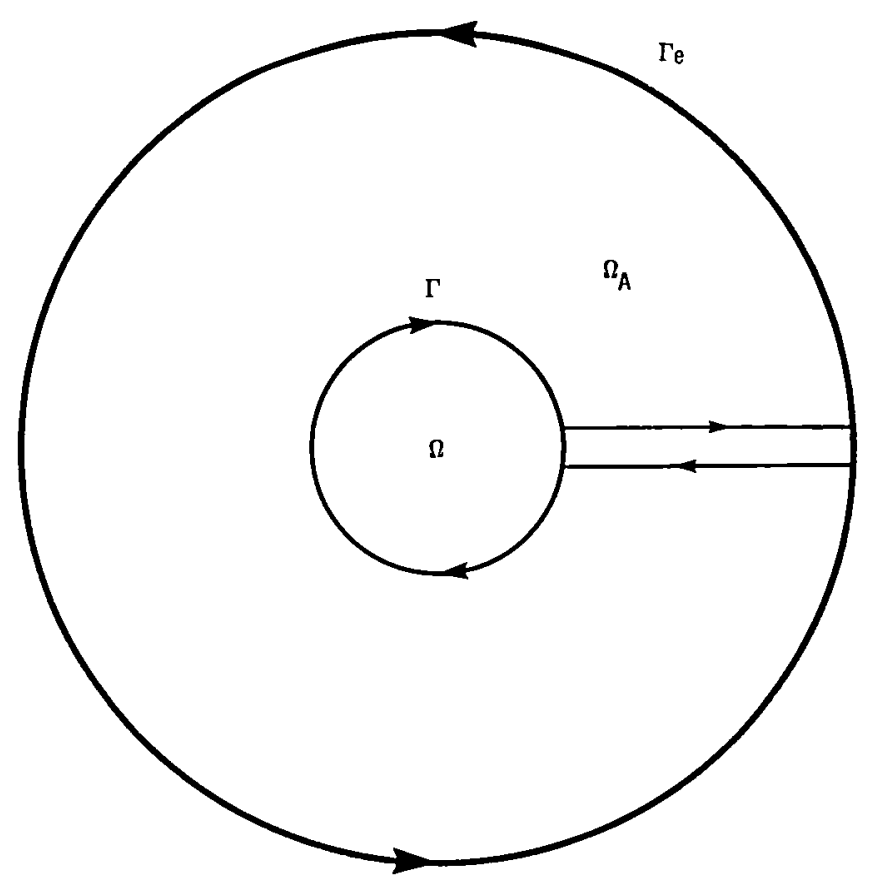

FIGURE 25. - COMPUTATIONAL DOMAIN FOR THE UNIQUENESS PROOF. 


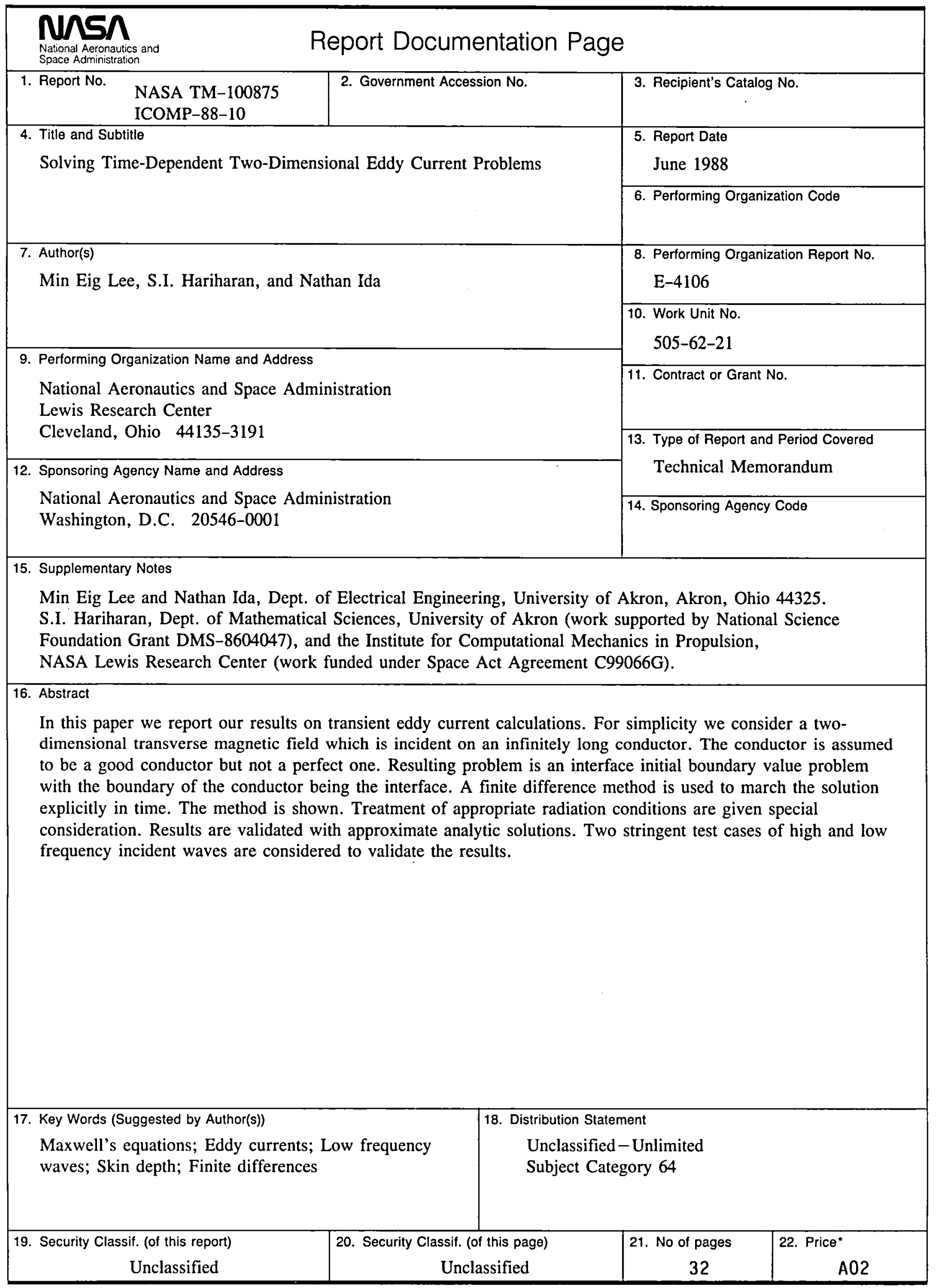


National Aeronautics and Space Administration

Lewis Research Center ICOMP (M.S. 5-3)

Cleveland, Ohio 44135

Otficial Business

Penalty for Private Use $\$ 300$
FOURTH CLASS MAIL

ADDRESS CORRECTION REQUESTED
|||||

Postage and Fees Paid

National Aeronaulics and

Space Admınıstration

NASA. 451 